\title{
Identification of 12 novel loci that confer susceptibility to early-onset dyslipidemia
}

\author{
YOSHIJI YAMADA ${ }^{1,2}$, KIMIHIKO KATO ${ }^{1,3}$, MITSUTOSHI OGURI $^{1,4}$, HIDEKI HORIBE $^{5}$, TETSUO FUJIMAKI ${ }^{6}$, \\ YOSHIKI YASUKOCHI $^{1,2}$, ICHIRO TAKEUCHI ${ }^{2,7,8}$ and JUN SAKUMA ${ }^{2,8,9}$
}

\begin{abstract}
${ }^{1}$ Department of Human Functional Genomics, Advanced Science Research Promotion Center, Mie University, Tsu, Mie 514-8507; ${ }^{2}$ CREST, Japan Science and Technology Agency, Kawaguchi, Saitama 332-0012; ${ }^{3}$ Department of Internal Medicine, Meitoh Hospital, Nagoya, Aichi 465-0025; ${ }^{4}$ Department of Cardiology, Kasugai Municipal Hospital, Kasugai, Aichi 486-8510; ${ }^{5}$ Department of Cardiovascular Medicine, Gifu Prefectural Tajimi Hospital, Tajimi, Gifu 507-8522; ${ }^{6}$ Department of Cardiovascular Medicine, Northern Mie Medical Center Inabe General Hospital, Inabe, Mie 511-0428; ${ }^{7}$ Department of Computer Science, Nagoya Institute of Technology, Nagoya, Aichi 466-8555; ${ }^{8}$ RIKEN Center for Advanced Intelligence Project, Tokyo 103-0027; ${ }^{9}$ Computer Science Department, College of Information Science, University of Tsukuba, Tsukuba, Ibaraki 305-8573, Japan
\end{abstract}

Received April 26, 2018; Accepted September 26, 2018

DOI: $10.3892 / \mathrm{ijmm} .2018 .3943$

\begin{abstract}
The circulating concentrations of triglycerides, high density lipoprotein (HDL)-cholesterol, and low density lipoprotein (LDL)-cholesterol have a substantial genetic component, and the heritability of early-onset dyslipidemia might be expected to be higher compared with late-onset forms. In the present study, exome-wide association studies (EWASs) were performed for early-onset hypertriglyceridemia, hypo-HDL-cholesterolemia, and hyper-LDL-cholesterolemia, with the aim to identify genetic variants that confer susceptibility to these conditions in the Japanese population. A total of 8,073 individuals aged $\leq 65$ years were enrolled in the study. The EWASs for hypertriglyceridemia $(2,664$ cases and 5,294 controls), hypo-HDL-cholesterolemia (974 cases and 7,085 controls), and hyper-LDL-cholesterolemia (2,911 cases and 5,111 controls) were performed with Illumina Human Exome-12 v1.2 DNA Analysis BeadChip or Infinium Exome-24 v1.0 BeadChip arrays. The association of allele frequencies for $31,198,31,133$, or 31,175 single nucleotide polymorphisms (SNPs) to hypertriglyceridemia, hypo-HDL-cholesterolemia, or hyper-LDL-cholesterolemia, respectively, was examined with Fisher's exact test. To compensate for multiple comparisons of genotypes with each of the three conditions, Bonferroni's correction was applied
\end{abstract}

Correspondence to: Professor Yoshiji Yamada, Department of Human Functional Genomics, Advanced Science Research Promotion Center, Mie University, 1577 Kurima-machiya, Tsu, Mie 514-8507, Japan

E-mail: yamada@gene.mie-u.ac.jp

Key words: dyslipidemia, hypercholesterolemia, hypertriglyceridemia, genetics, exome-wide association study for statistical significance of association. The results demonstrated that 25, 28 and 65 SNPs were significantly associated with hypertriglyceridemia, hypo-HDL-cholesterolemia and hyper-LDL-cholesterolemia, respectively. Multivariable logistic regression analysis with adjustment for age and sex revealed that all 25, 28 and 65 of these SNPs were significantly associated with hypertriglyceridemia, hypo-HDL-cholesterolemia and hyper-LDL-cholesterolemia, respectively. Following examination of the association of the identified SNPs to serum concentrations of triglycerides, HDL-cholesterol, or LDL-cholesterol, linkage disequilibrium of the SNPs, and results of previous genome-wide association studies, we newly identified chromosomal region $19 \mathrm{p} 12$ as a susceptibility locus for hypertriglyceridemia, eight loci (MOB3C-TMOD4, LPGAT1, EHD3, COL6A3, ZNF860-CACNA1D, COL6A5, $D C L R E 1 C, Z N F 77)$ for hypo-HDL-cholesterolemia, and three loci (KIAA0319-FAM65B, UBD, LOC105375015) for hyper-LDL-cholesterolemia. The present study thus identified 12 novel loci that may confer susceptibility to early-onset dyslipidemia. Determination of genotypes for the SNPs at these loci may prove informative for assessment of genetic risk for hypertriglyceridemia, hypo-HDL-cholesterolemia, or hyper-LDL-cholesterolemia in the Japanese population.

\section{Introduction}

Dyslipidemia, including hypertriglyceridemia, hypo-high density lipoprotein (HDL)-cholesterolemia and hyper-low density lipoprotein (LDL)-cholesterolemia, has a substantial genetic component (1-3). Familial hypercholesterolemia is an autosomal dominant disorder characterized by pronounced increases in the circulating concentrations of total cholesterol and LDL-cholesterol $(1,2)$. One of the underlying causes of familial hypercholesterolemia is a defect in the LDL receptor that is responsible for the uptake of most circulating LDL-cholesterol by the liver $(2,4)$. In addition to mutations 
of the LDL receptor gene $(L D L R)$, familial hypercholesterolemia can be caused by mutations in the apolipoprotein $\mathrm{B}$ gene $(A P O B)$, proprotein convertase subtilisin/kexin type 9 gene (PCSK9), cytochrome P450 family 7 subfamily A member 1 gene (CYP7A1), and LDL receptor adaptor protein 1 gene $(L D L R A P I)(2,4)$. Common forms of dyslipidemia are multifactorial and polygenic disorders, that result from an interaction between genetic background and both lifestyle and environmental factors, such as consumption of high-fat or high-calorie diets and physical inactivity $(1,5)$. The heritability of plasma concentrations of triglycerides, HDL-cholesterol, or LDL-cholesterol was demonstrated to be 33-43, 40-74, and $41-59 \%$, respectively (6-8). Given that dyslipidemia is a key risk factor for coronary artery disease and ischemic stroke $(9,10)$, as well as for colorectal cancer $(11,12)$, personalized prevention is an important public health goal.

Genome-wide association studies (GWASs) and gene-centric meta-analyses have implicated various genes and loci as determinants of blood lipid levels or of predisposition to dyslipidemia in European-ancestry populations (13-17). Genetic variants associated with lipid profiles have been extensively investigated, with one recent study having identified 157 such loci, including 62 variants not previously reported (18). Recent GWASs $(19,20)$ or studies based on whole-exome (21) or whole-genome (22) sequencing in European-ancestry populations have also identified low-frequency or rare variants associated with circulating lipid levels. A more recent exome-wide association study (EWAS) identified 444 independent variants at 250 loci as being significantly associated with plasma levels of total cholesterol, LDL-cholesterol, HDL-cholesterol, or triglycerides (23). An electronic health record-based study reported that genetic risk scores for circulating LDL-cholesterol and triglyceride levels based on 477 single nucleotide polymorphisms (SNPs) were predictive of age at initiation of treatment with lipid-lowering medication (24). Although various SNPs have been demonstrated to be associated with blood lipid profiles in East Asian $(25,26)$ or Japanese (27) populations, genetic variants that contribute to susceptibility to dyslipidemia in Japanese remain to be identified definitively.

Given the substantial genetic component of the circulating concentrations of triglycerides, HDL-cholesterol, and LDL-cholesterol (6-8), the genetic contribution to early-onset forms of hypertriglyceridemia, hypo-HDL-cholesterolemia, and hyper-LDL-cholesterolemia may be greater compared with late-onset forms $(2,3)$. The statistical power of genetic association studies may thus be increased by focusing on subjects with early-onset forms of these disorders.

The present study performed EWASs for early-onset forms of hypertriglyceridemia, hypo-HDL-cholesterolemia, and hyper-LDL-cholesterolemia with the use of human exome array-based genotyping methods. The aim was to identify genetic variants that confer susceptibility to these conditions in the Japanese population.

\section{Materials and methods}

Study subjects. In our previous studies, the median age of subjects with hypertriglyceridemia, hypo-HDL-cholesterolemia or hyper-LDL-cholesterolemia was 64,68 or 62 years, respectively (28). Therefore, in the present study, early-onset dyslipidemia was defined as that occurring at an age of $\leq 65$ years. A total of 8,073 individuals aged $\leq 65$ years were examined. Recruited subjects either visited outpatient clinics or were admitted to participating hospitals in Japan (Gifu Prefectural Tajimi Hospital, Tajimi; Gifu Prefectural General Medical Center, Gifu; Japanese Red Cross Nagoya First Hospital, Nagoya; Northern Mie Medical Center Inabe General Hospital, Inabe; Hirosaki University Hospital and Hirosaki Stroke and Rehabilitation Center, Hirosaki) because of various symptoms or for an annual health checkup between 2002 and 2014, or were community-dwelling individuals recruited to a population-based cohort study in Inabe between 2010 and 2014 (29).

Venous blood was collected in the early morning after subjects had fasted overnight. The blood samples were centrifuged at $1,600 \mathrm{x}$ g for $15 \mathrm{~min}$ at $4^{\circ} \mathrm{C}$, and serum was separated for subsequent analysis. Serum concentrations of triglycerides, HDL-cholesterol, and LDL-cholesterol were measured in the clinical laboratory of each hospital. The 2,664 subjects with hypertriglyceridemia and 5,294 controls had serum triglyceride concentrations of $\geq 1.69 \mathrm{mmol} / \mathrm{l}(150 \mathrm{mg} / \mathrm{dl})$ and $<1.69 \mathrm{mmol} / \mathrm{l}$, respectively. The 974 subjects with hypo-HDL-cholesterolemia and 7,085 controls had serum HDL-cholesterol concentrations of $<1.03 \mathrm{mmol} / 1(40 \mathrm{mg} / \mathrm{dl})$ and $\geq 1.03 \mathrm{mmol} / 1$, respectively. The 2,911 subjects with hyper-LDL-cholesterolemia and 5,111 controls had serum LDL-cholesterol concentrations of $\geq 3.62 \mathrm{mmol} / 1(140 \mathrm{mg} / \mathrm{dl})$ and $<3.62 \mathrm{mmol} / \mathrm{l}$, respectively. The 562 subjects with both hypertriglyceridemia and hypo-HDL-cholesterolemia, and the 4,907 controls, overlapped between the corresponding studies, as did the 1,312 subjects with both hypertriglyceridemia and hyper-LDL-cholesterolemia and 3,723 controls, as well as the 317 subjects with both hypo-HDL-cholesterolemia and hyper-LDL-cholesterolemia and 4,484 controls. Individuals with single-gene disorders, such as familial hypercholesterolemia, or with endocrinologic or metabolic diseases that cause dyslipidemia were excluded from the study. Those taking medications that may cause secondary dyslipidemia were also excluded.

EWAS. Venous blood was collected into tubes containing $50 \mathrm{mmol} / 1$ EDTA (disodium salt), peripheral blood leukocytes were isolated, and genomic DNA was extracted from these cells with the use of a kit (Genomix, Talent Srl, Trieste, Italy; or SMITEST EX-R\&D, Medical \& Biological Laboratories, Co., Ltd., Nagoya, Japan). EWASs for hypertriglyceridemia (2,664 cases and 5,294 controls), hypo-HDL-cholesterolemia (974 cases and 7,085 controls), and hyper-LDL-cholesterolemia (2,911 cases and 5,111 controls) were performed with Human Exome-12 v1.2 DNA Analysis BeadChip or Infinium Exome-24 v1.0 BeadChip arrays (Illumina, San Diego, CA, USA). These exome arrays include putative functional exonic variants selected from $\sim 12,000$ individual exome and whole-genome sequences. The exonic content consists of 244,000 SNPs from diverse populations, including European, African, Chinese, and Hispanic individuals (30). SNPs contained in only one of the arrays ( $\sim 2.6 \%$ of all SNPs) were excluded from analysis. Quality control was performed, as previously described (31). Briefly, genotyping data with a call rate of $<97 \%$ were discarded, with the mean call rate for 
Table I. Characteristics of subjects with hypertriglyceridemia and control individuals.

\begin{tabular}{|c|c|c|c|}
\hline Characteristic & Control & Hypertriglyceridemia & P-value \\
\hline Number of subjects & 5,294 & 2,664 & \\
\hline Age (years) & $51.3 \pm 10.2$ & $53.3 \pm 8.8$ & $<0.0001$ \\
\hline Sex (men/women, \%) & $50.2 / 49.8$ & $75.6 / 24.4$ & $<0.0001$ \\
\hline Smoking (\%) & 35.9 & 54.0 & $<0.0001$ \\
\hline Obesity (\%) & 22.9 & 42.6 & $<0.0001$ \\
\hline Body mass index $\left(\mathrm{kg} / \mathrm{m}^{2}\right)$ & $22.7 \pm 3.4$ & $24.8 \pm 3.6$ & $<0.0001$ \\
\hline Hypertension (\%) & 35.3 & 57.2 & $<0.0001$ \\
\hline Systolic BP (mmHg) & $125 \pm 23$ & $132 \pm 23$ & $<0.0001$ \\
\hline Diastolic BP (mmHg) & $75 \pm 14$ & $80 \pm 13$ & $<0.0001$ \\
\hline Diabetes mellitus (\%) & 17.5 & 33.8 & $<0.0001$ \\
\hline Fasting plasma glucose (mmol/l) & $5.83 \pm 2.04$ & $6.55 \pm 2.81$ & $<0.0001$ \\
\hline Blood hemoglobin $\mathrm{A}_{1 \mathrm{c}}(\%)$ & $5.83 \pm 1.08$ & $6.26 \pm 1.50$ & $<0.0001$ \\
\hline Serum triglycerides $(\mathrm{mmol} / \mathrm{l})$ & $0.94 \pm 0.34$ & $2.40 \pm 1.37$ & $<0.0001$ \\
\hline Serum HDL-cholesterol (mmol/l) & $1.66 \pm 0.47$ & $1.30 \pm 0.36$ & $<0.0001$ \\
\hline Serum LDL-cholesterol (mmol/l) & $3.06 \pm 0.80$ & $3.35 \pm 0.95$ & 0.8593 \\
\hline Chronic kidney disease (\%) & 12.3 & 19.9 & $<0.0001$ \\
\hline Serum creatinine $(\mu \mathrm{mol} / \mathrm{l})$ & $71.9 \pm 69.8$ & $82.1 \pm 85.2$ & $<0.0001$ \\
\hline eGFR (ml min $\left.{ }^{-1} 1.73 \mathrm{~m}^{-2}\right)$ & $78.5 \pm 19.4$ & $74.1 \pm 21.6$ & $<0.0001$ \\
\hline Hyperuricemia (\%) & 11.7 & 28.4 & $<0.0001$ \\
\hline Serum uric acid $(\mu \mathrm{mol} / \mathrm{l})$ & $308 \pm 90$ & $362 \pm 91$ & $<0.0001$ \\
\hline
\end{tabular}

Quantitative data are presented as mean \pm standard deviation and were compared between subjects with hypertriglyceridemia and controls with the unpaired Student's t-test. Categorical data were compared between the two groups with Pearson's Chi-square test. Based on Bonferroni's correction, $\mathrm{P}<0.0026(0.05 / 19)$ was considered statistically significant. BP, blood pressure; HDL, high density lipoprotein; LDL, low density lipoprotein; eGFR, estimated glomerular filtration rate.

the remaining data being $99.9 \%$. Gender specification was checked for all samples, and those for which gender phenotype in the clinical records was inconsistent with genetic sex were discarded. Duplicated samples and cryptic relatedness were checked by calculation of identity by descent; all pairs with a value of $>0.1875$ were inspected and one sample from each pair was excluded. Heterozygosity of SNPs was calculated for all samples, and those with extremely low or high heterozygosity (>3 standard deviations from the mean) were discarded. SNPs in sex chromosomes or mitochondrial DNA were excluded from analysis, as were non-polymorphic SNPs or SNPs with a minor allele frequency of $<1.0 \%$. SNPs whose genotype distributions deviated significantly $(\mathrm{P}<0.01)$ from Hardy-Weinberg equilibrium in control individuals were discarded. Finally, genotype data for each EWAS were examined for population stratification by principal components analysis (32), and population outliers were excluded from further study. A total of $31,198,31,133$, and 31,175 SNPs passed quality control in the EWASs for hypertriglyceridemia, hypo-HDL-cholesterolemia and hyper-LDL-cholesterolemia, respectively, and were subjected to further analyses.

Statistical analysis. For analysis of characteristics of the study subjects, quantitative and categorical data were compared between cases and controls with the unpaired Student's t-test and Pearson's Chi-square test, respectively. Allele frequencies were estimated by the gene counting method, and depar- ture from Hardy-Weinberg equilibrium was identified with Fisher's exact test. The association of allele frequencies of SNPs to hypertriglyceridemia, hypo-HDL-cholesterolemia or hyper-LDL-cholesterolemia in the EWASs was examined with Fisher's exact test. To compensate for multiple comparisons of allele frequencies with hypertriglyceridemia, hypo-HDL-cholesterolemia, or hyper-LDL-cholesterolemia, Bonferroni's correction was applied for statistical significance of association. Given that $31,198,31,133$ or 31,175 SNPs were analyzed, a P-value of $<1.60 \times 10^{-6}[0.05 /(31,198$ or 31,175$)]$ for hypertriglyceridemia and hyper-LDL-cholesterolemia and a P-value of $<1.61 \times 10^{-6}(0.05 / 31,133)$ for hypo-HDL-cholesterolemia was considered statistically significant for association. The inflation factor $(\lambda)$ was 1.07 for hypertriglyceridemia, 1.05 for hypo-HDL-cholesterolemia, and 1.00 for hyper-LDL-cholesterolemia. Multivariable logistic regression analysis was performed with hypertriglyceridemia, hypo-HDL-cholesterolemia, or hyper-LDL-cholesterolemia as a dependent variable and independent variables including age, sex (0, woman; 1 , man), and genotype of each SNP. Genotypes of SNPs were assessed according to dominant [0, $A A ; 1, A B+$ $B B$ ( $A$, major allele; $B$, minor allele $)]$, recessive $(0, A A+A B$; $1, B B)$, and additive genetic models, and the P-value, odds ratio, and $95 \%$ confidence interval were calculated. Additive models comprised additive $1(0, A A ; 1, A B ; 0, B B)$ and additive $2(0, A A ; 0, A B ; 1, B B)$ scenarios, which were analyzed simultaneously with a single statistical model. Associations 
Table II. Characteristics of subjects with hypo-HDL-cholesterolemia and control individuals.

\begin{tabular}{lccr}
\hline Characteristic & Control & Hypo-HDL-cholesterolemia & P-value \\
\hline Number of subjects & 7,085 & 974 & \\
Age (years) & $51.7 \pm 9.9$ & $54.3 \pm 8.7$ & $<0.0001$ \\
Sex (men/women, \%) & $55.1 / 44.9$ & $85.3 / 14.7$ & $<0.0001$ \\
Smoking (\%) & 40.5 & 54.6 & $<0.0001$ \\
Obesity (\%) & 27.0 & 48.2 & $<0.0001$ \\
Body mass index $\left(\mathrm{kg} / \mathrm{m}^{2}\right)$ & $23.2 \pm 3.5$ & $25.1 \pm 3.9$ & $<0.0001$ \\
Hypertension $(\%)$ & 39.6 & 64.8 & $<0.0001$ \\
Systolic BP $(\mathrm{mmHg})$ & $126 \pm 23$ & $137 \pm 27$ & $<0.0001$ \\
Diastolic BP $(\mathrm{mmHg})$ & $76 \pm 14$ & $80 \pm 16$ & $<0.0001$ \\
Diabetes mellitus $(\%)$ & 19.5 & 48.2 & $<0.0001$ \\
Fasting plasma glucose $(\mathrm{mmol} / \mathrm{l})$ & $5.93 \pm 2.16$ & $7.16 \pm 3.26$ & $<0.0001$ \\
Blood hemoglobin $\mathrm{A}_{\mathrm{lc}}(\%)$ & $5.89 \pm 1.17$ & $6.59 \pm 1.60$ & $<0.0001$ \\
Serum triglycerides $(\mathrm{mmol} / \mathrm{l})$ & $1.35 \pm 0.96$ & $2.12 \pm 1.62$ & $<0.0001$ \\
Serum HDL-cholesterol $(\mathrm{mmol} / \mathrm{l})$ & $1.63 \pm 0.42$ & $0.88 \pm 0.12$ & $<0.0001$ \\
Serum LDL-cholesterol $(\mathrm{mmol} / \mathrm{l})$ & $3.16 \pm 0.84$ & $3.13 \pm 0.99$ & 0.2860 \\
Chronic kidney disease $(\%)$ & 13.4 & 26.2 & $<0.0001$ \\
Serum creatinine $(\mu \mathrm{mol} / \mathrm{l})$ & $72.6 \pm 66.6$ & $96.0 \pm 122.7$ & $<0.0001$ \\
eGFR (ml min $\left.{ }^{-1} 1.73 \mathrm{~m}{ }^{-2}\right)$ & $77.5 \pm 18.5$ & $73.3 \pm 29.8$ & $<0.0001$ \\
Hyperuricemia $(\%)$ & 15.9 & 27.2 & $<0.0001$ \\
Serum uric acid $(\mu \mathrm{mol} / \mathrm{l})$ & $323 \pm 91$ & $361 \pm 0.3$ & $<0.0001$ \\
\hline
\end{tabular}

Quantitative data are presented as mean \pm standard deviation and were compared between subjects with hypo-HDL-cholesterolemia and controls with the unpaired Student's t-test. Categorical data were compared between the two groups with Pearson's Chi-square test. Based on Bonferroni's correction, $\mathrm{P}<0.0026(0.05 / 19)$ was considered statistically significant. BP, blood pressure; HDL, high density lipoprotein; LDL, low density lipoprotein; eGFR, estimated glomerular filtration rate.

of genotypes of identified SNPs to serum concentrations of triglycerides, HDL-cholesterol, or LDL-cholesterol were examined by one-way analysis of variance. Bonferroni's correction was also applied to other statistical analysis as indicated. Statistical tests were performed with JMP Genomics version 9.0 software (SAS Institute, Cary, NC, USA).

\section{Results}

Characteristics of subjects. The characteristics of the 7,958 subjects enrolled in the hypertriglyceridemia study are listed in Table I. Age, the frequency of men, and the prevalence of smoking, obesity, hypertension, diabetes mellitus, chronic kidney disease, and hyperuricemia as well as body mass index (BMI), systolic and diastolic blood pressure (BP), fasting plasma glucose (FPG) level, blood glycosylated hemoglobin (hemoglobin $\mathrm{A}_{1 \mathrm{c}}$ ) content, and serum concentrations of creatinine and uric acid were greater, whereas the serum concentration of HDL-cholesterol and estimated glomerular filtration rate (eGFR) were lower, in subjects with hypertriglyceridemia compared with controls.

The characteristics of the 8,059 subjects enrolled in the hypo-HDL-cholesterolemia study are listed in Table II. Age, the frequency of men, and the prevalence of smoking, obesity, hypertension, diabetes mellitus, chronic kidney disease, and hyperuricemia as well as BMI, systolic and diastolic BP, FPG level, blood hemoglobin $\mathrm{A}_{1 \mathrm{c}}$ content, and serum concentrations of triglycerides, creatinine, and uric acid were greater, whereas eGFR was lower, in subjects with hypo-HDL-cholesterolemia compared with controls.

The characteristics of the 8,022 subjects enrolled in the hyper-LDL-cholesterolemia study are listed in Table III. Age, the prevalence of obesity, BMI, diastolic BP, and serum concentrations of triglycerides and uric acid were greater, whereas systolic BP and serum concentrations of HDL-cholesterol and creatinine were lower, in subjects with hyper-LDL-cholesterolemia compared with controls.

EWASs for hypertriglyceridemia, hypo-HDL-cholesterolemia, and hyper-LDL-cholesterolemia. The association of allele frequencies for 31,198 SNPs that passed quality control to hypertriglyceridemia was examined with the use of Fisher's exact test. Following Bonferroni's correction, 25 SNPs were significantly associated with hypertriglyceridemia ( $\mathrm{P}<1.60 \times 10^{-6}$; Table IV). Similar analysis of the association of allele frequencies for 31,133 SNPs to hypo-HDL-cholesterolemia or of those for 31,175 SNPs to hyper-LDL-cholesterolemia revealed that 28 SNPs were significantly associated with hypo-HDL-cholesterolemia $\left(\mathrm{P}<1.61 \times 10^{-6}\right.$; Table $\left.\mathrm{V}\right)$ and 65 SNPs with hyper-LDL-cholesterolemia $\left(\mathrm{P}<1.60 \times 10^{-6}\right.$; Table VI).

Multivariable logistic regression analysis of the association of SNPs to hypertriglyceridemia, hypo-HDL-cholesterolemia or hyper-LDL-cholesterolemia. The association of the 
Table III. Characteristics of subjects with hyper-LDL-cholesterolemia and control individuals.

\begin{tabular}{|c|c|c|c|}
\hline Characteristic & Control & Hyper-LDL-cholesterolemia & P-value \\
\hline Number of subjects & 5,111 & 2,911 & \\
\hline Age (years) & $51.3 \pm 10.3$ & $53.2 \pm 8.9$ & $<0.0001$ \\
\hline Sex (men/women, \%) & $59.0 / 41.0$ & $58.2 / 41.8$ & 0.4936 \\
\hline Smoking $(\%)$ & 40.9 & 44.3 & 0.0034 \\
\hline Obesity (\%) & 27.7 & 32.6 & $<0.0001$ \\
\hline Body mass index $\left(\mathrm{kg} / \mathrm{m}^{2}\right)$ & $23.2 \pm 3.6$ & $23.9 \pm 3.5$ & $<0.0001$ \\
\hline Hypertension $(\%)$ & 42.5 & 42.6 & 0.8966 \\
\hline Systolic BP (mmHg) & $128 \pm 25$ & $126 \pm 21$ & 0.0025 \\
\hline Diastolic BP (mmHg) & $76 \pm 14$ & $77 \pm 13$ & 0.0004 \\
\hline Diabetes mellitus (\%) & 23.2 & 22.0 & 0.2593 \\
\hline Fasting plasma glucose (mmol/l) & $6.11 \pm 2.49$ & $6.02 \pm 2.12$ & 0.0831 \\
\hline Blood hemoglobin $\mathrm{A}_{1 \mathrm{c}}(\%)$ & $5.96 \pm 1.25$ & $5.99 \pm 1.26$ & 0.4573 \\
\hline Serum triglycerides $(\mathrm{mmol} / \mathrm{l})$ & $1.40 \pm 1.19$ & $1.52 \pm 0.91$ & $<0.0001$ \\
\hline Serum HDL-cholesterol (mmol/l) & $1.56 \pm 0.49$ & $1.51 \pm 0.42$ & $<0.0001$ \\
\hline Serum LDL-cholesterol (mmol/l) & $2.70 \pm 0.56$ & $3.90 \pm 0.74$ & $<0.0001$ \\
\hline Chronic kidney disease (\%) & 15.3 & 14.3 & 0.2118 \\
\hline Serum creatinine $(\mu \mathrm{mol} / \mathrm{l})$ & $78.3 \pm 88.7$ & $71.7 \pm 58.9$ & 0.0001 \\
\hline eGFR (ml min $\left.{ }^{-1} 1.73 \mathrm{~m}^{-2}\right)$ & $77.0 \pm 21.7$ & $76.8 \pm 17.8$ & 0.7007 \\
\hline Hyperuricemia (\%) & 16.3 & 18.9 & 0.0028 \\
\hline Serum uric acid $(\mu \mathrm{mol} / \mathrm{l})$ & $323 \pm 97$ & $334 \pm 87$ & $<0.0001$ \\
\hline
\end{tabular}

Quantitative data are presented as mean \pm standard deviation and were compared between subjects with hyper-LDL-cholesterolemia and controls with the unpaired Student's t-test. Categorical data were compared between the two groups with Pearson's Chi-square test. Based on Bonferroni's correction, $\mathrm{P}<0.0026(0.05 / 19)$ was considered statistically significant. BP, blood pressure; HDL, high density lipoprotein; LDL, low density lipoprotein; eGFR, estimated glomerular filtration rate.

25 SNPs identified in the EWAS for hypertriglyceridemia to this condition was further examined by multivariable logistic regression analysis, following adjustment for age and sex. All 25 SNPs were significantly $[\mathrm{P}<0.0005(0.05 / 100)$ in at least one genetic model] associated with hypertriglyceridemia (Table VII). Similar analysis revealed that all 28 SNPs identified in the EWAS for hypo-HDL-cholesterolemia $[\mathrm{P}<0.0004$ (0.05/112); Table VIII] and all 65 SNPs identified in the EWAS for hyper-LDL-cholesterolemia $[\mathrm{P}<0.0002$ $(0.05 / 260)$; Table IX] were significantly associated with the respective conditions.

Association of identified SNPs to serum concentrations of triglycerides, HDL-cholesterol or LDL-cholesterol. The association of the genotypes of identified SNPs to serum concentrations of triglycerides, HDL-cholesterol, or LDL-cholesterol was examined by one-way analysis of variance. The 25 SNPs identified in the EWAS for hypertriglyceridemia were all significantly associated with serum triglyceride concentration $[\mathrm{P}<0.0020(0.05 / 25)$; Table X]. Among the 28 SNPs identified in the EWAS for hypo-HDL-cholesterolemia, 27 polymorphisms were significantly associated with the serum HDL-cholesterol level $[\mathrm{P}<0.0018(0.05 / 28)]$, whereas rs114501427 of SPOPL was not related to this parameter (Table XI). Among the 65 SNPs identified in the EWAS for hyper-LDL-cholesterolemia, 37 SNPs were significantly associated with serum LDL-cholesterol concentration $[\mathrm{P}<0.0008(0.05 / 65)$; Table XII]. It is possible that the lack of significant correlation between the remaining 28 SNPs and the serum LDL-cholesterol levels may be due to effects of medical treatment.

Linkage disequilibrium analysis. The linkage disequilibrium (LD) was assessed among SNPs associated with hypertriglyceridemia, hypo-HDL-cholesterolemia, or hyper-LDL-cholesterolemia. For the hypertriglyceridemia study, strong LD was apparent among rs1260326 and rs780093 of GCKR and rs1260333 at chromosome 2p23 [square of the correlation coefficient $\left.\left(r^{2}\right), 0.876-0.983\right]$. An LD plot for the 12 SNPs located at chromosomal region $8 \mathrm{p} 21.3$ is illustrated in Fig. 1. Strong LD was observed among rs301, rs326, rs13702, and rs15285 of $L P L$, rs2083637 and rs1441756 ( $\left.r^{2}, 0.942-0.988\right)$, as well as among rs328 of $L P L$, rs10096633, rs17482753, rs12678919, rs10503669 and rs7016880 ( $\left.r^{2}, 0.910-0.999\right)$. An LD plot for the eight SNPs located at chromosomal region 11q23.3 is illustrated in Fig. 2. Significant LD was apparent among rs10790162 of $B U D 13$, rs7350481, rs9326246, both rs964184 and rs2075290 of ZPR1, and rs2266788 of APOA5 $\left(r^{2}, 0.687-0.990\right)$.

For the hypo-HDL-cholesterolemia study, significant LD was apparent between rs139537100 of MOB3C and rs115287176 of TMOD4 $\left(r^{2}, 0.984\right)$; between rs116417209 of EHD3 and rs114501427 of SPOPL $\left(r^{2}, 0.703\right)$; between rs140232911 of ZNF860 and rs35874056 of CACNA1D 
Table IV. The 25 SNPs significantly associated with hypertriglyceridemia in the exome-wide association study.

\begin{tabular}{|c|c|c|c|c|c|c|c|c|}
\hline Gene & SNP & $\begin{array}{l}\text { Nucleotide } \\
\text { substitution }^{\text {a }}\end{array}$ & $\begin{array}{l}\text { Amino acid } \\
\text { substitution }\end{array}$ & Chromosome & Position & MAF (\%) & Allele OR & $\begin{array}{c}\text { Allele } \\
\text { frequency }(\mathrm{P} \text {-value })\end{array}$ \\
\hline APOA5 & rs2075291 & $\mathrm{G} / \mathrm{T}$ & G185C & 11 & 116790676 & 7.3 & 1.89 & $2.83 \times 10^{-24}$ \\
\hline$B U D 13$ & rs10790162 & $\mathrm{G} / \mathrm{A}$ & & 11 & 116639104 & 26.3 & 1.47 & $3.58 \times 10^{-24}$ \\
\hline ZPRI & rs964184 & $\mathrm{C} / \mathrm{G}$ & & 11 & 116778201 & 26.3 & 1.46 & $8.82 \times 10^{-24}$ \\
\hline \multirow[t]{3}{*}{$A P O A 5$} & rs2266788 & $\mathrm{T} / \mathrm{C}$ & & 11 & 116789970 & 26.2 & 1.47 & $1.27 \times 10^{-23}$ \\
\hline & rs7350481 & $\mathrm{C} / \mathrm{T}$ & & 11 & 116715567 & 27.7 & 1.44 & $7.94 \times 10^{-23}$ \\
\hline & rs9326246 & $\mathrm{G} / \mathrm{C}$ & & 11 & 116741017 & 26.5 & 1.45 & $1.36 \times 10^{-22}$ \\
\hline \multirow[t]{5}{*}{ ZPRI } & rs2075290 & $\mathrm{T} / \mathrm{C}$ & & 11 & 116782580 & 26.7 & 1.43 & $1.58 \times 10^{-21}$ \\
\hline & rs12678919 & $\mathrm{A} / \mathrm{G}$ & & 8 & 19986711 & 12.6 & 0.71 & $6.07 \times 10^{-11}$ \\
\hline & rs10503669 & $\mathrm{C} / \mathrm{A}$ & & 8 & 19990179 & 12.6 & 0.71 & $8.32 \times 10^{-11}$ \\
\hline & rs17482753 & $\mathrm{G} / \mathrm{T}$ & & 8 & 19975135 & 12.6 & 0.72 & $2.04 \times 10^{-10}$ \\
\hline & rs10096633 & $\mathrm{C} / \mathrm{T}$ & & 8 & 19973410 & 12.7 & 0.72 & $2.50 \times 10^{-10}$ \\
\hline$L P L$ & rs328 & $\mathrm{C} / \mathrm{G}$ & $\mathrm{S} 474^{*}$ & 8 & 19962213 & 12.9 & 0.72 & $2.72 \times 10^{-10}$ \\
\hline \multirow[t]{2}{*}{ APOA4 } & rs5104 & $\mathrm{A} / \mathrm{G}$ & N147S & 11 & 116821618 & 35.7 & 1.25 & $2.78 \times 10^{-10}$ \\
\hline & rs7016880 & $\mathrm{G} / \mathrm{C}$ & & 8 & 20019235 & 12.0 & 0.72 & $1.72 \times 10^{-9}$ \\
\hline$G C K R$ & rs1260326 & $\mathrm{T} / \mathrm{C}$ & L446P & 2 & 27508073 & 43.6 & 0.82 & $6.27 \times 10^{-9}$ \\
\hline \multirow[t]{5}{*}{$G C K R$} & rs780093 & $\mathrm{A} / \mathrm{G}$ & & 2 & 27519736 & 43.0 & 0.83 & $2.18 \times 10^{-8}$ \\
\hline & rs1260333 & $\mathrm{T} / \mathrm{C}$ & & 2 & 27525757 & 42.9 & 0.83 & $4.38 \times 10^{-8}$ \\
\hline & rs11085421 & $\mathrm{A} / \mathrm{C}$ & & 19 & 20985948 & 19.1 & 0.79 & $1.59 \times 10^{-7}$ \\
\hline & rs1441756 & $\mathrm{T} / \mathrm{G}$ & & 8 & 20010875 & 19.0 & 0.80 & $5.61 \times 10^{-7}$ \\
\hline & rs2083637 & $\mathrm{T} / \mathrm{C}$ & & 8 & 20007664 & 19.0 & 0.81 & $6.24 \times 10^{-7}$ \\
\hline$L P L$ & rs301 & $\mathrm{T} / \mathrm{C}$ & & 8 & 19959423 & 19.3 & 0.81 & $7.70 \times 10^{-7}$ \\
\hline$L P L$ & rs13702 & $\mathrm{A} / \mathrm{G}$ & & 8 & 19966981 & 19.2 & 0.81 & $9.15 \times 10^{-7}$ \\
\hline \multirow[t]{2}{*}{$L P L$} & rs15285 & $\mathrm{G} / \mathrm{A}$ & & 8 & 19967156 & 19.2 & 0.81 & $1.02 \times 10^{-6}$ \\
\hline & rs2954033 & $\mathrm{G} / \mathrm{A}$ & & 8 & 125481504 & 33.3 & 1.19 & $1.02 \times 10^{-6}$ \\
\hline$L P L$ & rs326 & $\mathrm{A} / \mathrm{G}$ & & 8 & 19961928 & 19.4 & 0.81 & $1.24 \times 10^{-6}$ \\
\hline
\end{tabular}

Allele frequencies were analyzed with Fisher's exact test. $\mathrm{P}<1.60 \times 10^{-6}$ was considered statistically significant. ${ }^{a}$ Major allele/minor allele. SNP, single nucleotide polymorphism; MAF, minor allele frequency; OR, odds ratio.

$\left(r^{2}, 1.00\right) ;$ among rs192210727 of $A D G R L 3$, rs188378669 of CXCL8, and rs61734696 of MARCH1 ( $r^{2}, 0.901$ to 0.972$)$; between rs199921354 of VPS33B and rs141569282 of OR4F6 $\left(r^{2}, 0.994\right)$; and between rs247616 and rs3764261 at chromosome $16 \mathrm{q} 13\left(r^{2}, 0.992\right)$. An LD plot for the five SNPs located at chromosomal region $12 \mathrm{q} 24.12$ to $12 \mathrm{q} 24.13$ is illustrated in Fig. 3. Significant LD was detected among rs3782886 of BRAP, rs11066015 of $A C A D 10$, rs671 of $A L D H 2$, and rs2074356 and rs11066280 of HECTD4 $\left(r^{2}, 0.813-0.995\right)$.

For the hyper-LDL-cholesterolemia study, significant LD was apparent among rs147284320 of PTCH2, rs139537100 of MOB3C and rs115287176 of TMOD4 $\left(r^{2}, 0.828-0.984\right)$; among rs192210727 of ADGRL3, rs188378669 of CXCL8, and rs61734696 of MARCHI $\left(r^{2}, 0.901-0.972\right)$; and between rs200787930 of PLCB2 and rs199921354 of VPS33B $\left(r^{2}\right.$, 0.994). An LD plot for the 47 SNPs located at chromosomal region $6 \mathrm{p} 22.3$ to $6 \mathrm{p} 21.3$ is illustrated in Fig. 4 . There were two major LD blocks. The first LD block $\left(r^{2}, 0.571-1.000\right)$ comprised rs7749235, rs2285321, and rs6937357 of DHX16; rs9468805, rs6457254, and rs2394392 of PPP1R18; rs3130663 at $6 \mathrm{p} 21.3$; rs2269702, rs28986465, rs2075015, and rs6924270 of $M D C 1$; and rs3132584, rs25527, and rs9500864 of
TUBB. The second LD block $\left(r^{2}, 0.762-1.000\right)$ comprised rs147733073 of CCHCR1; rs2596574 at 6p21.3; rs11538264 of PRRC2A; rs9267546, rs17200983, and rs9267547 of LY6G6F; rs117894946 of LY6G6C; rs11754464 of MSH5; rs5030798 and rs11751198 of VARS; rs6457452 of HSPA1B; rs11968400 of C6orf48; rs12210887 at 6p21.3; rs13118 of NEU1; rs117127493 of SLC44A4; rs492899 of SKIV2L; rs140770834 and rs11751545 of TNXB; and rs204999 at 6p21.3. Significant LD was also observed between rs4576240 of KIAA0319 and rs150142878 of FAM65B ( $\left.r^{2}, 0.893\right)$; among rs34902660 of SLC17A3, rs76463649 of ZSCAN26, rs6922302 of ZSCAN31, rs29243 of GABBR1, and rs2523995 of TRIM40 $\left(r^{2}, 0.601-0.995\right)$; between rs64036 of $U B D$ and $\mathrm{rs} 2524272$ at $6 \mathrm{p} 22.1\left(r^{2}, 0.753\right)$; and among rs6933400 and rs11970154 of DPCR 1, rs 2508015 at $6 \mathrm{p} 21.3$, and rs147733073 of CCHCR 1 $\left(r^{2}, 0.596-0.987\right)$.

Relation of SNPs identified in the present study to dyslipidemia-related phenotypes examined in previous GWASs. The relation of genes, chromosomal loci and SNPs identified in the present study was further analyzed against phenotypes previously examined by GWASs available in the 
Table V. The 28 SNPs significantly associated with hypo-HDL-cholesterolemia in the exome-wide association study.

\begin{tabular}{|c|c|c|c|c|c|c|c|c|}
\hline Gene & SNP & $\begin{array}{l}\text { Nucleotide } \\
\text { substitution }^{\mathrm{a}}\end{array}$ & $\begin{array}{l}\text { Amino acid } \\
\text { substitution }\end{array}$ & Chromosome & Position & MAF (\%) & Allele OR & $\begin{array}{c}\text { Allele } \\
\text { frequency } \\
\text { (P-value) }\end{array}$ \\
\hline$L P G A T 1$ & rs150552771 & $\mathrm{T} / \mathrm{C}$ & K200E & 1 & 211783358 & 5.0 & 16.67 & $1.09 \times 10^{-12}$ \\
\hline AРOA5 & rs2075291 & $\mathrm{G} / \mathrm{T}$ & G185C & 11 & 116790676 & 7.3 & 1.79 & $4.10 \times 10^{-12}$ \\
\hline COL6A5 & rs200982668 & $\mathrm{G} / \mathrm{A}$ & $\mathrm{E} 2501 \mathrm{~K}$ & 3 & 130470894 & 1.3 & 0.18 & $4.51 \times 10^{-11}$ \\
\hline \multirow[t]{2}{*}{ ZNF860 } & rs140232911 & $\mathrm{C} / \mathrm{T}$ & S161L & 3 & 31989561 & 10.4 & 4.39 & $5.21 \times 10^{-11}$ \\
\hline & rs9261800 & $\mathrm{C} / \mathrm{G}$ & & 6 & 30408822 & 2.8 & 19.65 & $6.69 \times 10^{-11}$ \\
\hline$V P S 33 B$ & rs199921354 & $\mathrm{C} / \mathrm{T}$ & R80Q & 15 & 91013841 & 1.2 & 0.17 & $1.03 \times 10^{-10}$ \\
\hline ADGRL3 & rs192210727 & $\mathrm{G} / \mathrm{T}$ & R580I & 4 & 61909615 & 1.3 & 0.19 & $1.33 \times 10^{-10}$ \\
\hline TMOD4 & rs115287176 & $\mathrm{G} / \mathrm{A}$ & R277W & 1 & 151170961 & 1.2 & 0.18 & $1.46 \times 10^{-10}$ \\
\hline COL6A3 & rs146092501 & $\mathrm{C} / \mathrm{T}$ & E1386K & 2 & 237371861 & 1.2 & 0.18 & $2.10 \times 10^{-10}$ \\
\hline MARCHI & rs61734696 & $\mathrm{G} / \mathrm{T}$ & Q137K & 4 & 164197303 & 1.2 & 0.19 & $2.74 \times 10^{-10}$ \\
\hline PLCB2 & rs200787930 & $\mathrm{C} / \mathrm{T}$ & E1106K & 15 & 40289298 & 1.2 & 0.19 & $2.79 \times 10^{-10}$ \\
\hline$M O B 3 C$ & rs139537100 & $\mathrm{C} / \mathrm{T}$ & R24Q & 1 & 46615006 & 1.2 & 0.19 & $2.87 \times 10^{-10}$ \\
\hline$C X C L 8$ & rs188378669 & $\mathrm{G} / \mathrm{T}$ & E31* & 4 & 73741568 & 1.2 & 0.19 & $3.94 \times 10^{-10}$ \\
\hline EHD3 & rs116417209 & G/A & V151I & 2 & 31249417 & 3.5 & 12.5 & $5.66 \times 10^{-10}$ \\
\hline ZNF77 & rs146879198 & $\mathrm{G} / \mathrm{A}$ & R340* & 19 & 2934109 & 1.2 & 0.20 & $1.17 \times 10^{-9}$ \\
\hline OR4F6 & rs 141569282 & $\mathrm{G} / \mathrm{A}$ & A117T & 15 & 101806068 & 1.7 & 0.23 & $2.31 \times 10^{-9}$ \\
\hline$A C A D 10$ & rs11066015 & $\mathrm{G} / \mathrm{A}$ & & 12 & 111730205 & 27.5 & 1.37 & $2.61 \times 10^{-9}$ \\
\hline CACNAID & rs35874056 & $\mathrm{G} / \mathrm{A}$ & G460S & 3 & 53702798 & 2.0 & 100.00 & $2.95 \times 10^{-9}$ \\
\hline \multirow[t]{3}{*}{$A L D H 2$} & rs671 & $\mathrm{G} / \mathrm{A}$ & E504K & 12 & 111803962 & 27.6 & 1.35 & $4.23 \times 10^{-9}$ \\
\hline & rs3764261 & $\mathrm{G} / \mathrm{T}$ & & 16 & 56959412 & 19.8 & 0.69 & $7.90 \times 10^{-9}$ \\
\hline & rs247616 & $\mathrm{C} / \mathrm{T}$ & & 16 & 56955678 & 19.7 & 0.69 & $9.31 \times 10^{-9}$ \\
\hline$B R A P$ & rs3782886 & $\mathrm{A} / \mathrm{G}$ & & 12 & 111672685 & 29.3 & 1.34 & $1.19 \times 10^{-8}$ \\
\hline CETP & rs1532624 & $\mathrm{G} / \mathrm{T}$ & & 16 & 56971567 & 29.6 & 0.74 & $2.63 \times 10^{-8}$ \\
\hline HECTD4 & rs11066280 & $\mathrm{T} / \mathrm{A}$ & & 12 & 112379979 & 29.0 & 1.33 & $3.54 \times 10^{-8}$ \\
\hline HECTD4 & rs2074356 & $\mathrm{C} / \mathrm{T}$ & & 12 & 112207597 & 25.4 & 1.32 & $1.43 \times 10^{-7}$ \\
\hline$L I L R B 2$ & rs73055442 & $\mathrm{C} / \mathrm{T}$ & $\mathrm{R} 103 \mathrm{H}$ & 19 & 54279838 & 1.6 & 34.09 & $1.80 \times 10^{-7}$ \\
\hline DCLRE1C & rs150854849 & $\mathrm{C} / \mathrm{T}$ & R179Q & 10 & 14934704 & 2.4 & 59.56 & $3.16 \times 10^{-7}$ \\
\hline$S P O P L$ & rs114501427 & $\mathrm{G} / \mathrm{A}$ & D349N & 2 & 138568946 & 8.8 & 3.23 & $4.01 \times 10^{-7}$ \\
\hline
\end{tabular}

Allele frequencies were analyzed with Fisher's exact test. $\mathrm{P}<1.61 \times 10^{-6}$ was considered statistically significant. ${ }^{a}$ Major allele/minor allele. SNP, single nucleotide polymorphism; MAF, minor allele frequency; OR, odds ratio.

Genome-Wide Repository of Associations Between SNPs and Phenotypes (GRASP) Search v.2.0.0.0 (https://grasp. nhlbi.nih.gov/Search.aspx) developed by the Information Technology and Applications Center, National Center for Biotechnology Information, National Heart, Lung, and Blood Institute, National Institutes of Health (Bethesda, MD, USA) $(33,34)$.

In the hypertriglyceridemia study, $G C K R$, chromosomal region $2 \mathrm{p} 23, L P L$, region $8 \mathrm{p} 21.3$, region $8 \mathrm{q} 24.2, B U D 13$, region 11q23.3, ZPR1, APOA5, and APOA4 have been previously reported to be associated with circulating triglyceride concentrations, whereas chromosome $19 \mathrm{p} 12$ had not been related to triglyceride levels or other dyslipidemia-related traits (Table XIII).

In the study of hypo-HDL-cholesterolemia, APOA5, $B R A P, A L D H 2, H E C T D 4$, chromosome 16q13, CETP, and $L I L R B 2$ were previously reported to be associated with circulating HDL-cholesterol levels, whereas CXCL8, ACAD10, and $P L C B 2$ were associated with circulating levels of total cholesterol, LDL-cholesterol, and triglycerides, respectively (Table XIV). MARCH1, chromosome 6p22.1, and VPS33B were also associated with adiponectin concentrations, type 1 diabetes mellitus, and type 2 diabetes mellitus, respectively. The remaining 13 genes (MOB3C, TMOD4, LPGAT1, EHD3, SPOPL, COL6A3, ZNF860, CACNA1D, COL6A5, ADGRL3, DCLRE1C, OR4F6, ZNF77) had not been related to circulating HDL-cholesterol or other dyslipidemia-related phenotypes.

In the study of hyper-LDL-cholesterolemia, $P C S K 9, A P O B$, chromosome 6p21.3, SKIV2L, HECTD4, APOE, and APOC1 were previously reported to be associated with circulating LDL-cholesterol levels, whereas CXCL8, 6p21.3, CCHCR1, PRRC2A, LY6G6F, MSH5, C6orf48, SLC44A4, TNXB, and $P L C B 2$ were associated with circulating concentrations of total cholesterol or triglycerides (Table XV). MARCH1, SLC17A3, ZSCAN31, GABBR1, 6p22.1, TRIM40, PPP1R18, 6p21.3, $M D C 1, T U B B, D P C R 1, L Y 6 G 6 C, V A R S, H S P A 1 B$, and VPS33B 
Table VI. The 65 SNPs significantly associated with hyper-LDL-cholesterolemia in the exome-wide association study.

\begin{tabular}{|c|c|c|c|c|c|c|c|c|}
\hline Gene & SNP & $\begin{array}{l}\text { Nucleotide } \\
\text { substitution }^{\mathrm{a}}\end{array}$ & $\begin{array}{l}\text { Amino acid } \\
\text { substitution }\end{array}$ & Chromosome & Position & MAF (\%) & Allele OR & $\begin{array}{c}\text { Allele } \\
\text { frequency } \\
\text { (P-value) }\end{array}$ \\
\hline$A P O E$ & rs7412 & $\mathrm{C} / \mathrm{T}$ & $\mathrm{R} 176 \mathrm{C}$ & 19 & 44908822 & 4.3 & 0.42 & $6.62 \times 10^{-22}$ \\
\hline COL6A3 & rs146092501 & $\mathrm{C} / \mathrm{T}$ & E1386K & 2 & 237371861 & 1.2 & 2.14 & $1.42 \times 10^{-11}$ \\
\hline TMOD4 & rs115287176 & $\mathrm{G} / \mathrm{A}$ & R277W & 1 & 151170961 & 1.2 & 2.13 & $1.83 \times 10^{-11}$ \\
\hline CXCL8 & rs188378669 & $\mathrm{G} / \mathrm{T}$ & E31* & 4 & 73741568 & 1.2 & 2.10 & $1.95 \times 10^{-11}$ \\
\hline$V P S 33 B$ & rs199921354 & $\mathrm{C} / \mathrm{T}$ & R80Q & 15 & 91013841 & 1.2 & 2.11 & $2.32 \times 10^{-11}$ \\
\hline ZNF77 & rs146879198 & $\mathrm{G} / \mathrm{A}$ & R340* & 19 & 2934109 & 1.2 & 2.08 & $4.91 \times 10^{-11}$ \\
\hline$M O B 3 C$ & rs139537100 & $\mathrm{C} / \mathrm{T}$ & R24Q & 1 & 46615006 & 1.2 & 2.06 & $5.58 \times 10^{-11}$ \\
\hline$P L C B 2$ & rs200787930 & $\mathrm{C} / \mathrm{T}$ & E1106K & 15 & 40289298 & 1.2 & 2.03 & $1.70 \times 10^{-10}$ \\
\hline MUC17 & rs78010183 & $\mathrm{A} / \mathrm{T}$ & $\mathrm{T} 1305 \mathrm{~S}$ & 7 & 101035329 & 1.8 & 1.81 & $2.30 \times 10^{-10}$ \\
\hline$M A R C H 1$ & rs61734696 & $\mathrm{G} / \mathrm{T}$ & Q137K & 4 & 164197303 & 1.2 & 2.01 & $3.49 \times 10^{-10}$ \\
\hline$A P O B$ & rs13306206 & $\mathrm{G} / \mathrm{A}$ & P955S & 2 & 21019859 & 3.2 & 1.75 & $7.32 \times 10^{-10}$ \\
\hline COL6A5 & rs200982668 & $\mathrm{G} / \mathrm{A}$ & $\mathrm{E} 2501 \mathrm{~K}$ & 3 & 130470894 & 1.3 & 1.92 & $9.85 \times 10^{-10}$ \\
\hline РTCH2 & rs147284320 & $\mathrm{C} / \mathrm{T}$ & V503I & 1 & 44828589 & 2.0 & 1.86 & $1.23 \times 10^{-9}$ \\
\hline$A D G R L 3$ & rs192210727 & $\mathrm{G} / \mathrm{T}$ & R580I & 4 & 61909615 & 1.3 & 1.94 & $1.49 \times 10^{-9}$ \\
\hline$A P O C 1$ & rs445925 & $\mathrm{C} / \mathrm{T}$ & & 19 & 44912383 & 6.6 & 0.66 & $3.62 \times 10^{-9}$ \\
\hline$H S P A 1 B$ & rs6457452 & $\mathrm{C} / \mathrm{T}$ & & 6 & 31827773 & 9.7 & 1.33 & $2.97 \times 10^{-8}$ \\
\hline \multirow[t]{2}{*}{ C6orf48 } & rs11968400 & $\mathrm{C} / \mathrm{T}$ & & 6 & 31836952 & 9.7 & 1.33 & $3.96 \times 10^{-8}$ \\
\hline & rs12210887 & $\mathrm{G} / \mathrm{T}$ & & 6 & 31847946 & 9.7 & 1.33 & $5.26 \times 10^{-8}$ \\
\hline$U B D$ & rs64036 & $\mathrm{C} / \mathrm{A}$ & & 6 & 29559490 & 22.5 & 1.23 & $7.55 \times 10^{-8}$ \\
\hline CCHCRI & rs147733073 & $\mathrm{C} / \mathrm{G}$ & H539Q & 6 & 31145462 & 10.2 & 1.32 & $8.01 \times 10^{-8}$ \\
\hline \multirow[t]{2}{*}{$V A R S$} & rs11751198 & $\mathrm{G} / \mathrm{A}$ & & 6 & 31785749 & 9.5 & 1.33 & $8.12 \times 10^{-8}$ \\
\hline & rs2596574 & $\mathrm{G} / \mathrm{A}$ & & 6 & 31366397 & 9.7 & 1.32 & $8.83 \times 10^{-8}$ \\
\hline$L Y 6 G 6 F$ & rs9267547 & $\mathrm{G} / \mathrm{A}$ & $\mathrm{A} 107 \mathrm{~T}$ & 6 & 31707724 & 10.0 & 1.32 & $9.72 \times 10^{-8}$ \\
\hline$N E U 1$ & rs13118 & $\mathrm{T} / \mathrm{A}$ & & 6 & 31859509 & 9.7 & 1.32 & $1.01 \times 10^{-7}$ \\
\hline SLC17A3 & rs34902660 & $\mathrm{C} / \mathrm{A}$ & G239V & 6 & 25850874 & 8.4 & 1.35 & $1.04 \times 10^{-7}$ \\
\hline FAM65B & rs 150142878 & $\mathrm{C} / \mathrm{T}$ & R371Q & 6 & 24847657 & 5.6 & 1.42 & $1.18 \times 10^{-7}$ \\
\hline \multirow[t]{3}{*}{$T U B B$} & rs9500864 & $\mathrm{G} / \mathrm{A}$ & & 6 & 30725455 & 20.4 & 1.23 & $1.19 \times 10^{-7}$ \\
\hline & rs204999 & $\mathrm{A} / \mathrm{G}$ & & 6 & 32142202 & 9.1 & 1.33 & $1.34 \times 10^{-7}$ \\
\hline & rs3130663 & $\mathrm{A} / \mathrm{G}$ & & 6 & 30698817 & 10.7 & 1.28 & $1.38 \times 10^{-7}$ \\
\hline$V A R S$ & rs5030798 & $\mathrm{G} / \mathrm{A}$ & V1055I & 6 & 31779733 & 9.5 & 1.32 & $1.43 \times 10^{-7}$ \\
\hline$L Y 6 G 6 F$ & rs9267546 & $\mathrm{G} / \mathrm{A}$ & & 6 & 31705659 & 9.8 & 1.32 & $1.44 \times 10^{-7}$ \\
\hline PRRC2A & rs11538264 & $\mathrm{G} / \mathrm{A}$ & V1774M & 6 & 31635412 & 9.5 & 1.32 & $1.55 \times 10^{-7}$ \\
\hline DPCRl & rs11970154 & $\mathrm{G} / \mathrm{C}$ & G1213R & 6 & 30952101 & 13.3 & 1.28 & $1.60 \times 10^{-7}$ \\
\hline MSH5 & rs11754464 & $\mathrm{C} / \mathrm{T}$ & & 6 & 31755958 & 9.5 & 1.32 & $1.73 \times 10^{-7}$ \\
\hline PCSK 9 & rs151193009 & $\mathrm{C} / \mathrm{T}$ & R93C & 1 & 55043912 & 1.1 & 0.42 & $1.85 \times 10^{-7}$ \\
\hline$G A B B R 1$ & rs29243 & $\mathrm{C} / \mathrm{T}$ & & 6 & 29631325 & 10.6 & 1.30 & $1.94 \times 10^{-7}$ \\
\hline KIAA0319 & rs4576240 & $\mathrm{G} / \mathrm{T}$ & P133T & 6 & 24596250 & 5.5 & 1.41 & $2.10 \times 10^{-7}$ \\
\hline$L Y 6 G 6 F$ & rs17200983 & $\mathrm{C} / \mathrm{A}$ & P34Q & 6 & 31707506 & 9.5 & 1.32 & $2.38 \times 10^{-7}$ \\
\hline$T N X B$ & rs140770834 & $\mathrm{C} / \mathrm{G}$ & L2271V & 6 & 32064851 & 8.8 & 1.32 & $2.42 \times 10^{-7}$ \\
\hline$T N X B$ & rs11751545 & $\mathrm{A} / \mathrm{C}$ & & 6 & 32073266 & 8.8 & 1.32 & $2.42 \times 10^{-7}$ \\
\hline$D P C R 1$ & rs6933400 & $\mathrm{C} / \mathrm{T}$ & & 6 & 30939399 & 13.2 & 1.27 & $2.43 \times 10^{-7}$ \\
\hline$L Y 6 G 6 C$ & rs117894946 & $\mathrm{G} / \mathrm{C}$ & G75A & 6 & 31719250 & 9.5 & 1.32 & $2.66 \times 10^{-7}$ \\
\hline SLC44A4 & rs117127493 & $\mathrm{G} / \mathrm{C}$ & E344Q & 6 & 31869232 & 8.9 & 1.32 & $3.22 \times 10^{-7}$ \\
\hline ZSCAN26 & rs76463649 & $\mathrm{A} / \mathrm{G}$ & N15S & 6 & 28271963 & 9.6 & 1.30 & $4.26 \times 10^{-7}$ \\
\hline DHX16 & rs7749235 & $\mathrm{T} / \mathrm{C}$ & & 6 & 30667816 & 17.8 & 1.23 & $4.67 \times 10^{-7}$ \\
\hline $\begin{array}{l}\text { LOC 105- } \\
375015\end{array}$ & rs9264942 & $\mathrm{T} / \mathrm{C}$ & & 6 & 31306603 & 40.1 & 1.18 & $4.82 \times 10^{-7}$ \\
\hline ZSCAN31 & rs6922302 & $\mathrm{C} / \mathrm{G}$ & P128A & 6 & 28327533 & 9.6 & 1.30 & $5.06 \times 10^{-7}$ \\
\hline$M D C 1$ & rs2269702 & $\mathrm{A} / \mathrm{G}$ & & 6 & 30707358 & 17.7 & 1.23 & $5.13 \times 10^{-7}$ \\
\hline$M D C 1$ & rs28986465 & $\mathrm{C} / \mathrm{T}$ & P386L & 6 & 30712785 & 17.7 & 1.23 & $5.17 \times 10^{-7}$ \\
\hline
\end{tabular}


Table VI. Continued.

\begin{tabular}{|c|c|c|c|c|c|c|c|c|}
\hline Gene & SNP & $\begin{array}{l}\text { Nucleotide } \\
\text { substitution }^{\mathrm{a}}\end{array}$ & $\begin{array}{l}\text { Amino acid } \\
\text { substitution }\end{array}$ & Chromosome & Position & MAF (\%) & Allele OR & $\begin{array}{c}\text { Allele } \\
\text { frequency } \\
(\mathrm{P} \text {-value })\end{array}$ \\
\hline$M D C 1$ & rs2075015 & $\mathrm{G} / \mathrm{A}$ & E371K & 6 & 30712831 & 17.7 & 1.23 & $5.17 \times 10^{-7}$ \\
\hline DHX16 & rs2285321 & $\mathrm{T} / \mathrm{C}$ & & 6 & 30670221 & 17.8 & 1.23 & $5.18 \times 10^{-7}$ \\
\hline SKIV2L & rs492899 & $\mathrm{A} / \mathrm{G}$ & & 6 & 31965741 & 9.1 & 1.31 & $5.33 \times 10^{-7}$ \\
\hline \multirow[t]{2}{*}{$M D C 1$} & rs6924270 & $\mathrm{A} / \mathrm{G}$ & & 6 & 30714203 & 17.8 & 1.23 & $5.81 \times 10^{-7}$ \\
\hline & rs2508015 & $\mathrm{C} / \mathrm{T}$ & & 6 & 31042423 & 11.7 & 1.27 & $6.07 \times 10^{-7}$ \\
\hline$T U B B$ & rs25527 & $\mathrm{C} / \mathrm{T}$ & & 6 & 30723161 & 21.0 & 1.22 & $6.08 \times 10^{-7}$ \\
\hline PPP1R18 & rs9468805 & $\mathrm{G} / \mathrm{A}$ & & 6 & 30675932 & 17.7 & 1.23 & $7.07 \times 10^{-7}$ \\
\hline DHX16 & rs6937357 & $\mathrm{T} / \mathrm{C}$ & & 6 & 30672547 & 17.8 & 1.23 & $7.07 \times 10^{-7}$ \\
\hline HECTD4 & rs11066280 & $\mathrm{T} / \mathrm{A}$ & & 12 & 112379979 & 29.0 & 1.19 & $7.09 \times 10^{-7}$ \\
\hline TRIM40 & rs2523995 & $\mathrm{G} / \mathrm{A}$ & & 6 & 30134407 & 10.6 & 1.28 & $7.88 \times 10^{-7}$ \\
\hline PPP1R18 & rs6457254 & $\mathrm{C} / \mathrm{T}$ & & 6 & 30681357 & 17.9 & 1.22 & $1.20 \times 10^{-6}$ \\
\hline \multirow[t]{2}{*}{$T U B B$} & rs3132584 & $\mathrm{C} / \mathrm{A}$ & & 6 & 30720650 & 21.2 & 1.20 & $1.22 \times 10^{-6}$ \\
\hline & rs3130685 & $\mathrm{T} / \mathrm{C}$ & & 6 & 31238429 & 41.6 & 0.85 & $1.43 \times 10^{-6}$ \\
\hline GIT2 & rs2292354 & $\mathrm{C} / \mathrm{T}$ & & 12 & 109930396 & 26.2 & 0.83 & $1.45 \times 10^{-6}$ \\
\hline \multirow[t]{2}{*}{ PPPIR18 } & rs2394392 & $\mathrm{T} / \mathrm{G}$ & & 6 & 30682541 & 18.0 & 1.22 & $1.50 \times 10^{-6}$ \\
\hline & rs 2524272 & $\mathrm{~T} / \mathrm{C}$ & & 6 & 29714623 & 26.8 & 1.19 & $1.55 \times 10^{-6}$ \\
\hline
\end{tabular}

Allele frequencies were analyzed with Fisher's exact test. $\mathrm{P}<1.60 \times 10^{-6}$ was considered statistically significant. ${ }^{\mathrm{a}}$ Major allele/minor allele. SNP, single nucleotide polymorphism; MAF, minor allele frequency; OR, odds ratio.

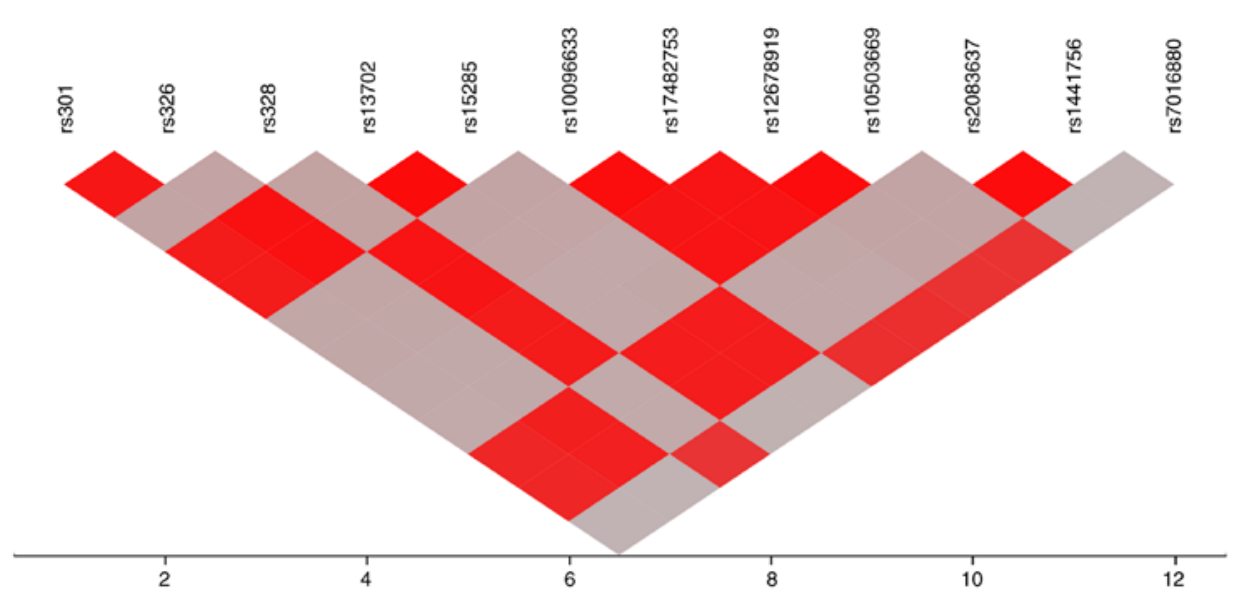

Figure 1. LD map for 12 SNPs at chromosomal region 8p21.3 associated with hypertriglyceridemia. LD was calculated as the square of the correlation coefficient $\left(r^{2}\right)$, with the extent of LD increasing according to the color order of gray to red. LD, linkage disequilibrium; SNP, single nucleotide polymorphism.

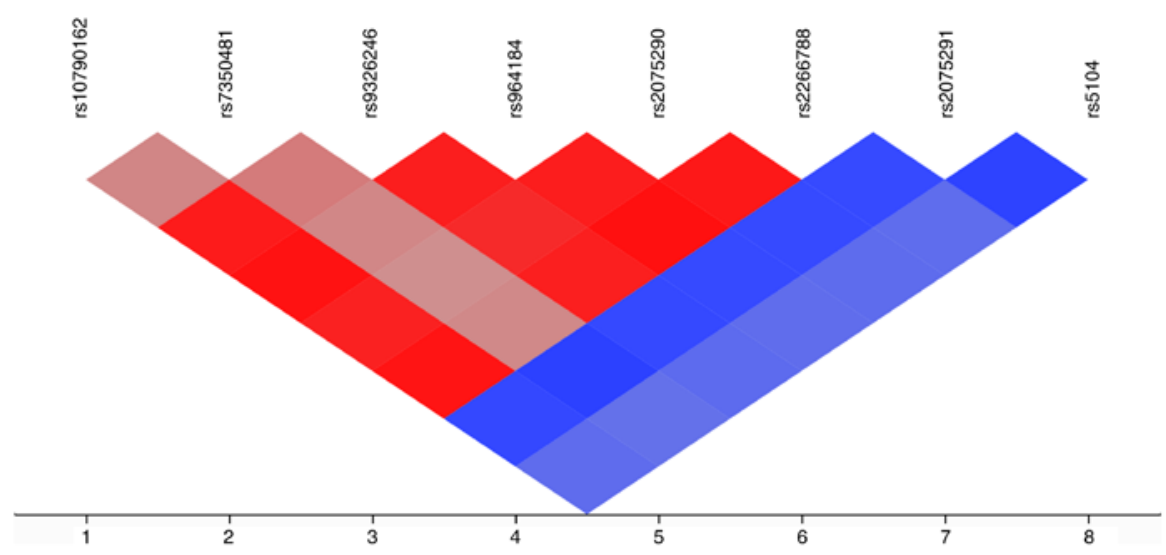

Figure 2. LD map for eight SNPs at chromosomal region 11q23.3 associated with hypertriglyceridemia. LD was calculated as the square of the correlation coefficient $\left(r^{2}\right)$, with the extent of LD increasing according to the color order of blue to gray to red. LD, linkage disequilibrium; SNP, single nucleotide polymorphism. 


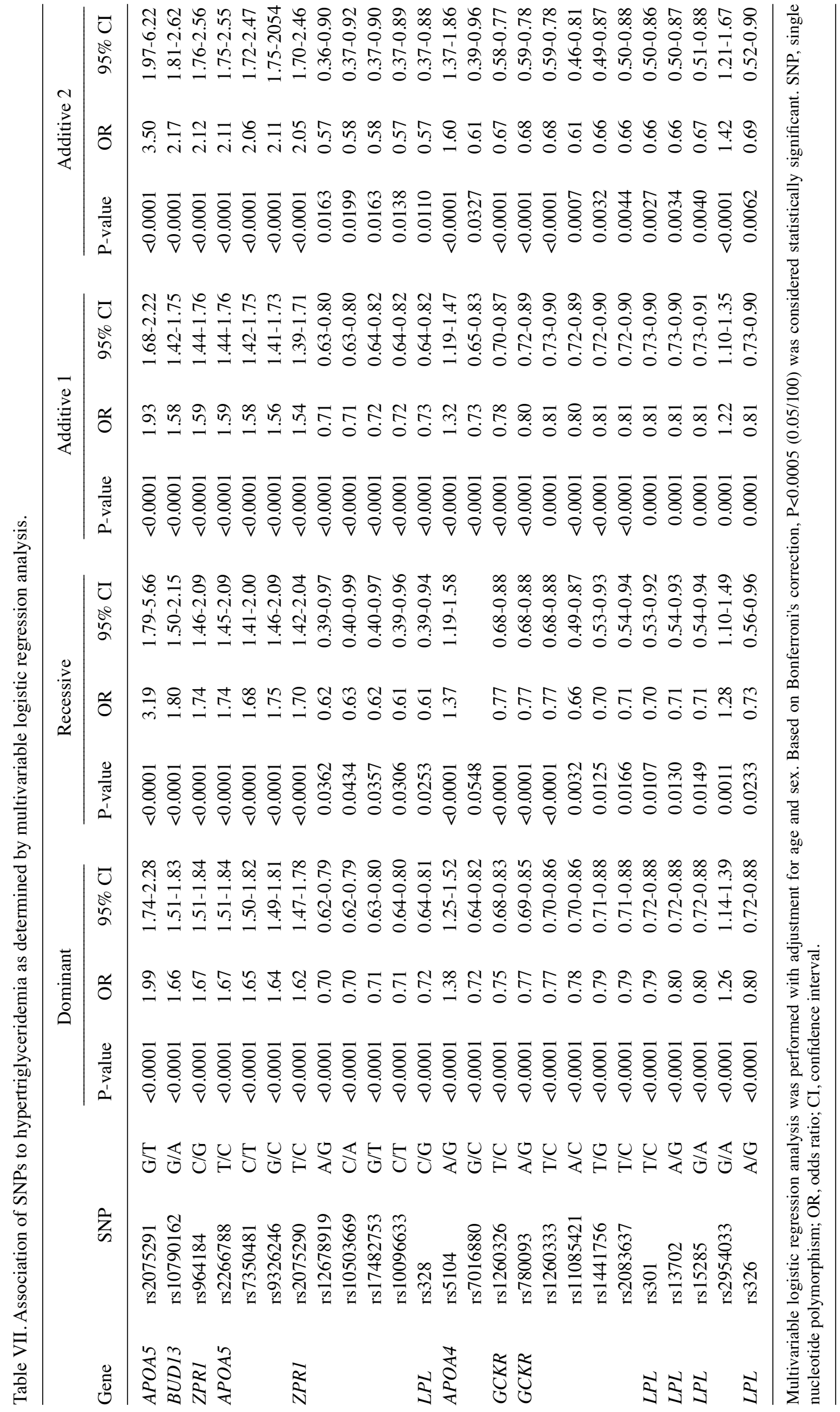




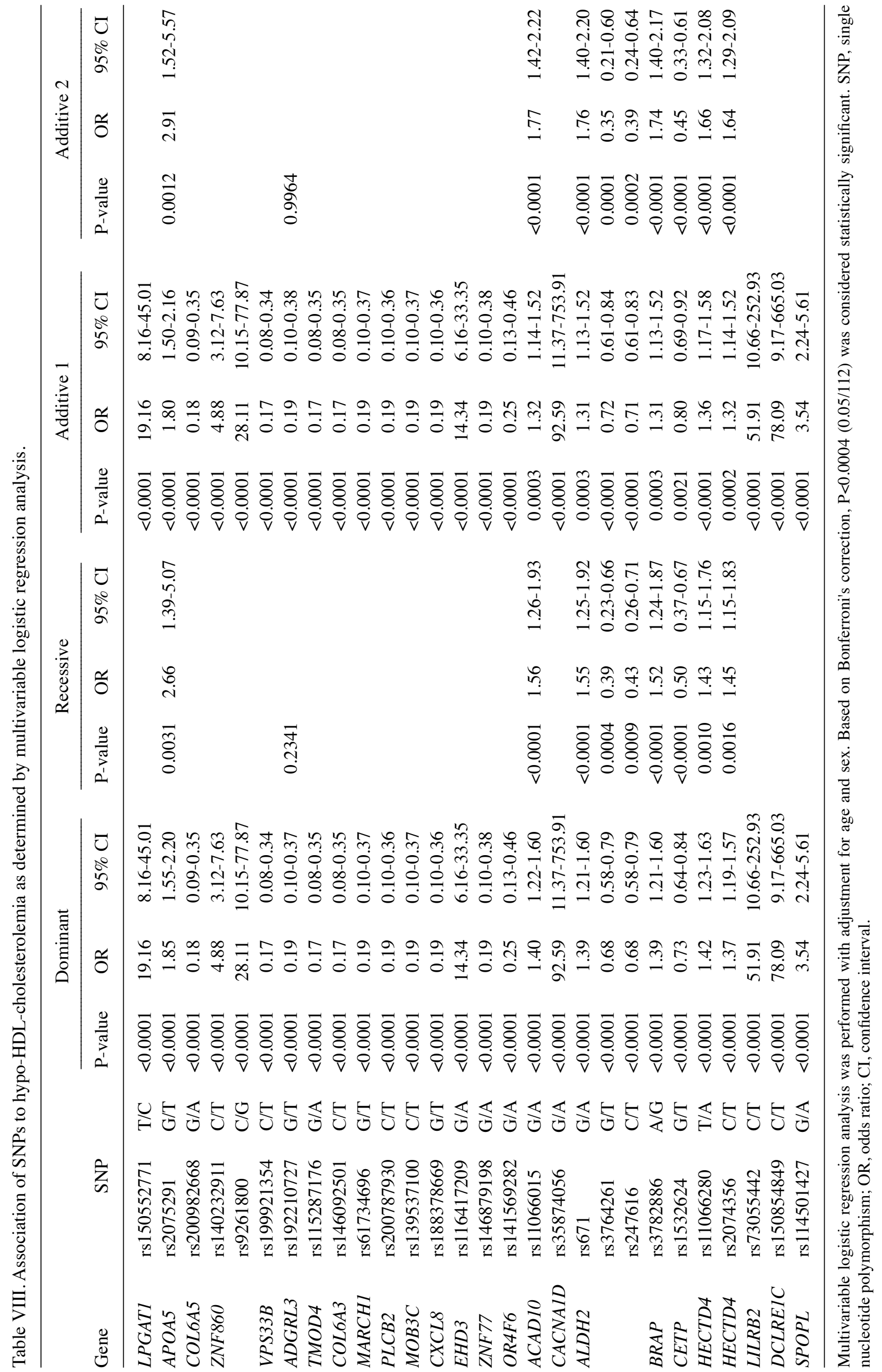




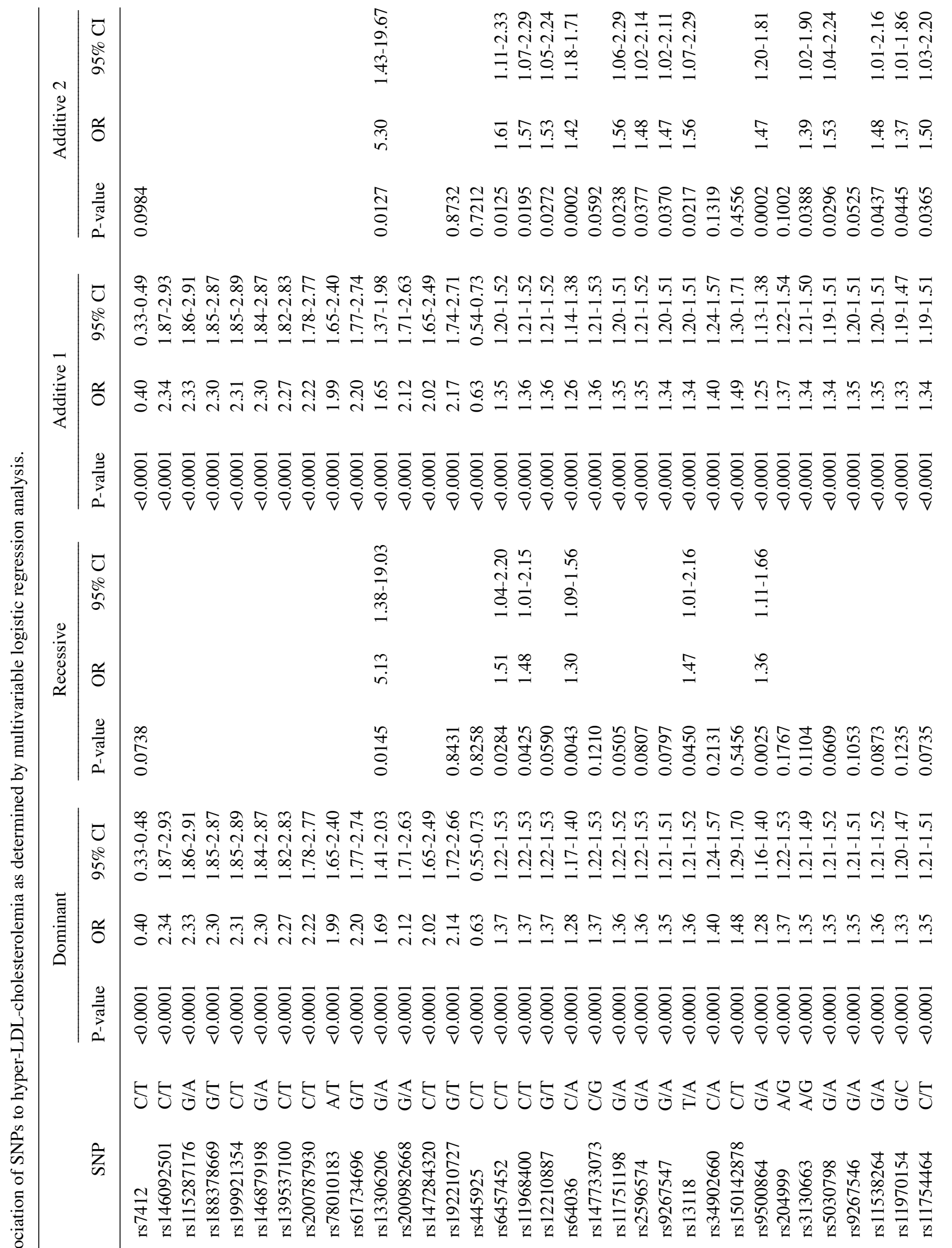




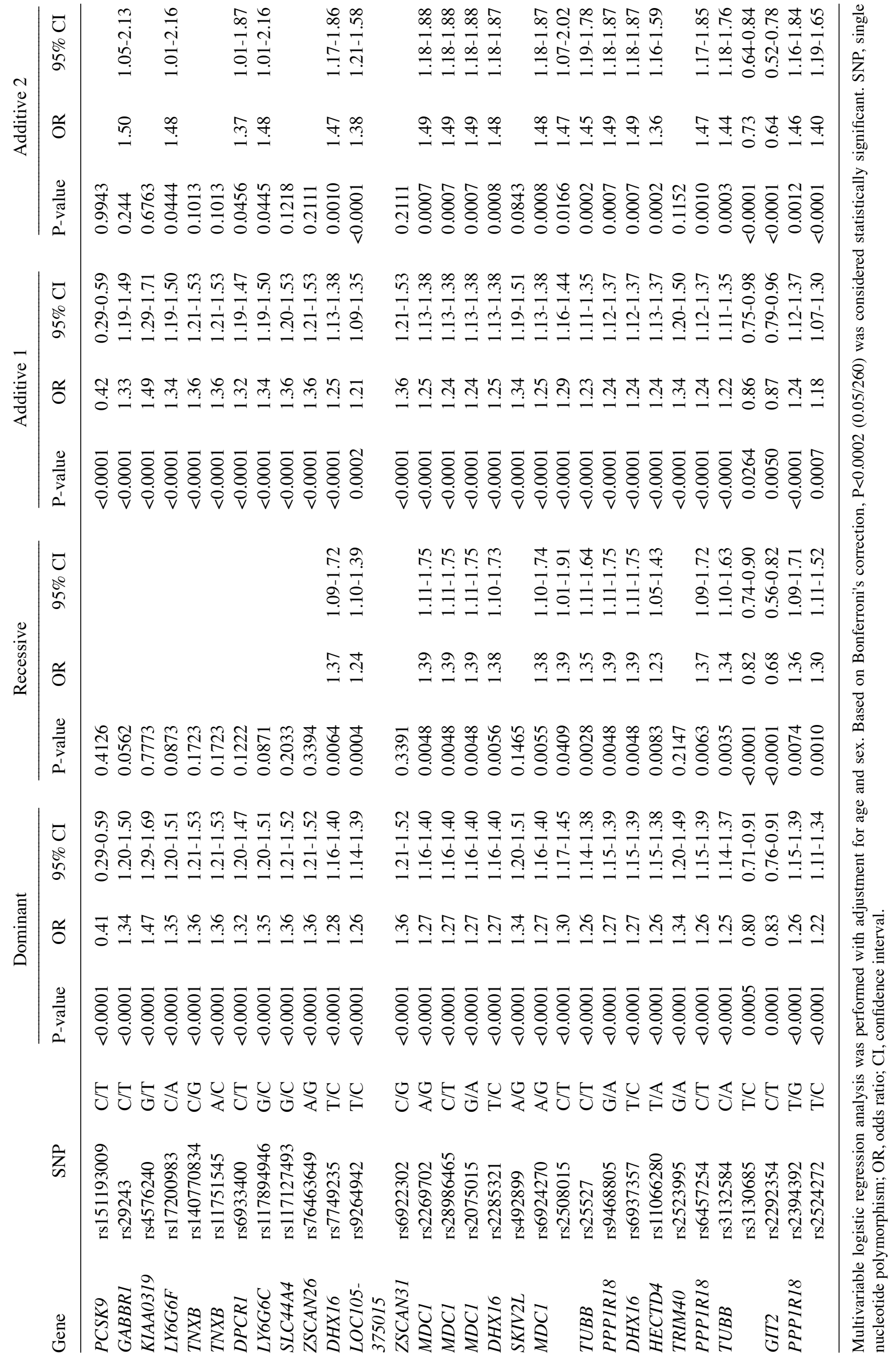


Table X. Association of SNPs identified in the present study to serum triglyceride concentration.

\begin{tabular}{|c|c|c|c|c|c|c|}
\hline \multirow{3}{*}{$\frac{\text { Gene }}{A P O A 5}$} & \multicolumn{2}{|c|}{ SNP } & \multicolumn{3}{|c|}{ Serum triglycerides $(\mathrm{mmol} / \mathrm{l})$} & \multirow{3}{*}{$\begin{array}{c}\text { P-value } \\
<0.0001\end{array}$} \\
\hline & rs2075291 & $\mathrm{G} / \mathrm{T}$ & GG & GT & TT & \\
\hline & & & $1.39 \pm 0.98$ & $1.83 \pm 1.55$ & $2.56 \pm 2.27$ & \\
\hline \multirow[t]{2}{*}{ BUD13 } & rs10790162 & $\mathrm{G} / \mathrm{A}$ & GG & GA & AA & $<0.0001$ \\
\hline & & & $1.35 \pm 1.00$ & $1.54 \pm 1.18$ & $1.77 \pm 1.31$ & \\
\hline \multirow[t]{2}{*}{$Z P R 1$} & rs964184 & $\mathrm{C} / \mathrm{G}$ & $\mathrm{CC}$ & CG & GG & $<0.0001$ \\
\hline & & & $1.35 \pm 1.00$ & $1.55 \pm 1.18$ & $1.75 \pm 1.30$ & \\
\hline \multirow{6}{*}{ APOA5 } & rs2266788 & $\mathrm{T} / \mathrm{C}$ & $\mathrm{TT}$ & $\mathrm{TC}$ & $\mathrm{CC}$ & $<0.0001$ \\
\hline & & & $1.35 \pm 1.00$ & $1.54 \pm 1.17$ & $1.76 \pm 1.30$ & \\
\hline & rs7350481 & $\mathrm{C} / \mathrm{T}$ & $\mathrm{CC}$ & CT & TT & $<0.0001$ \\
\hline & & & $1.34 \pm 0.95$ & $1.56 \pm 1.24$ & $1.68 \pm 1.19$ & \\
\hline & rs9326246 & $\mathrm{G} / \mathrm{C}$ & GG & GC & $\mathrm{CC}$ & $<0.0001$ \\
\hline & & & $1.35 \pm 1.00$ & $1.54 \pm 1.18$ & $1.74 \pm 1.28$ & \\
\hline \multirow[t]{10}{*}{ ZPR1 } & rs2075290 & $\mathrm{T} / \mathrm{C}$ & TT & $\mathrm{TC}$ & $\mathrm{CC}$ & $<0.0001$ \\
\hline & & & $1.36 \pm 1.01$ & $1.54 \pm 1.17$ & $1.74 \pm 1.29$ & \\
\hline & rs12678919 & $\mathrm{A} / \mathrm{G}$ & $\mathrm{AA}$ & AG & GG & $<0.0001$ \\
\hline & & & $1.49 \pm 1.11$ & $1.33 \pm 1.03$ & $1.32 \pm 1.37$ & \\
\hline & rs10503669 & $\mathrm{C} / \mathrm{A}$ & $\mathrm{CC}$ & $\mathrm{CA}$ & AA & $<0.0001$ \\
\hline & & & $1.49 \pm 1.11$ & $1.33 \pm 1.03$ & $1.32 \pm 1.38$ & \\
\hline & rs17482753 & $\mathrm{G} / \mathrm{T}$ & GG & GT & TT & $<0.0001$ \\
\hline & & & $1.49 \pm 1.11$ & $1.33 \pm 1.02$ & $1.31 \pm 1.35$ & \\
\hline & rs 10096633 & $\mathrm{C} / \mathrm{T}$ & $\mathrm{CC}$ & $\mathrm{CT}$ & TT & $<0.0001$ \\
\hline & & & $1.49 \pm 1.11$ & $1.33 \pm 1.03$ & $1.31 \pm 1.34$ & \\
\hline \multirow[t]{2}{*}{$L P L$} & rs328 & $\mathrm{C} / \mathrm{G}$ & $\mathrm{CC}$ & CG & GG & $<0.0001$ \\
\hline & & & $1.49 \pm 1.11$ & $1.33 \pm 1.02$ & $1.33 \pm 1.33$ & \\
\hline \multirow[t]{4}{*}{ APOA4 } & rs5104 & $\mathrm{A} / \mathrm{G}$ & $\mathrm{AA}$ & $\mathrm{AG}$ & GG & $<0.0001$ \\
\hline & & & $1.37 \pm 1.00$ & $1.49 \pm 1.12$ & $1.57 \pm 1.28$ & \\
\hline & rs7016880 & $\mathrm{G} / \mathrm{C}$ & GG & GC & $\mathrm{CC}$ & $<0.0001$ \\
\hline & & & $1.49 \pm 1.11$ & $1.33 \pm 1.04$ & $1.30 \pm 1.35$ & \\
\hline GCKR & rs 1260326 & $\mathrm{~T} / \mathrm{C}$ & $\mathrm{TT}$ & $\mathrm{TC}$ & $\mathrm{CC}$ & $<0.0001$ \\
\hline & & & $1.54 \pm 1.14$ & $1.44 \pm 1.14$ & $1.33 \pm 0.89$ & \\
\hline \multirow[t]{10}{*}{ GCKR } & rs780093 & $\mathrm{A} / \mathrm{G}$ & AA & $\mathrm{AG}$ & GG & $<0.0001$ \\
\hline & & & $1.53 \pm 1.12$ & $1.45 \pm 1.16$ & $1.33 \pm 0.87$ & \\
\hline & rs 1260333 & $\mathrm{~T} / \mathrm{C}$ & $\mathrm{TT}$ & $\mathrm{TC}$ & $\mathrm{CC}$ & $<0.0001$ \\
\hline & & & $1.53 \pm 1.12$ & $1.45 \pm 1.16$ & $1.32 \pm 0.87$ & \\
\hline & rs11085421 & $\mathrm{A} / \mathrm{C}$ & AA & $\mathrm{AC}$ & $\mathrm{CC}$ & $<0.0001$ \\
\hline & & & $1.49 \pm 1.14$ & $1.39 \pm 1.05$ & $1.27 \pm 0.70$ & \\
\hline & rs 1441756 & $\mathrm{~T} / \mathrm{G}$ & $\mathrm{TT}$ & TG & GG & $<0.0001$ \\
\hline & & & $1.49 \pm 1.12$ & $1.38 \pm 1.04$ & $1.34 \pm 1.16$ & \\
\hline & rs2083637 & $\mathrm{T} / \mathrm{C}$ & $\mathrm{TT}$ & $\mathrm{TC}$ & $\mathrm{CC}$ & $<0.0001$ \\
\hline & & & $1.49 \pm 1.12$ & $1.38 \pm 1.05$ & $1.34 \pm 1.16$ & \\
\hline \multirow[t]{2}{*}{$L P L$} & rs301 & $\mathrm{T} / \mathrm{C}$ & $\mathrm{TT}$ & $\mathrm{TC}$ & $\mathrm{CC}$ & $<0.0001$ \\
\hline & & & $1.49 \pm 1.11$ & $1.38 \pm 1.08$ & $1.34 \pm 1.14$ & \\
\hline \multirow[t]{2}{*}{$L P L$} & rs13702 & $\mathrm{A} / \mathrm{G}$ & AA & $\mathrm{AG}$ & GG & $<0.0001$ \\
\hline & & & $1.50 \pm 1.12$ & $1.38 \pm 1.04$ & $1.34 \pm 1.15$ & \\
\hline \multirow[t]{4}{*}{$L P L$} & rs 15285 & $\mathrm{G} / \mathrm{A}$ & GG & GA & $\mathrm{AA}$ & $<0.0001$ \\
\hline & & & $1.50 \pm 1.12$ & $1.38 \pm 1.04$ & $1.34 \pm 1.15$ & \\
\hline & rs2954033 & $\mathrm{G} / \mathrm{A}$ & GG & GA & AA & 0.0007 \\
\hline & & & $1.41 \pm 1.02$ & $1.47 \pm 1.16$ & $1.55 \pm 1.18$ & \\
\hline \multirow[t]{2}{*}{$L P L$} & rs326 & $\mathrm{A} / \mathrm{G}$ & AA & $\mathrm{AG}$ & GG & $<0.0001$ \\
\hline & & & $1.49 \pm 1.11$ & $1.38 \pm 1.07$ & $1.35 \pm 1.14$ & \\
\hline
\end{tabular}

Data are presented as mean \pm standard deviation and were compared among genotypes by one-way analysis of variance. Based on Bonferroni's correction, $\mathrm{P}<0.0020(0.05 / 25)$ was considered statistically significant. SNP, single nucleotide polymorphism. 
Table XI. Association of SNPs identified in the present study to serum HDL-cholesterol concentration.

\begin{tabular}{|c|c|c|c|c|c|c|}
\hline \multirow{2}{*}{$\frac{\text { Gene }}{L P G A T 1}$} & \multicolumn{2}{|c|}{ SNP } & \multicolumn{3}{|c|}{ Serum HDL-cholesterol (mmol/l) } & \multirow{2}{*}{$\begin{array}{l}\text { P-value } \\
<0.0001\end{array}$} \\
\hline & rs 150552771 & $\mathrm{~T} / \mathrm{C}$ & TT & $\mathrm{TC}$ & & \\
\hline & & & $1.54 \pm 0.47$ & $1.11 \pm 0.63$ & & \\
\hline \multirow[t]{2}{*}{ APOA5 } & rs2075291 & $\mathrm{G} / \mathrm{T}$ & GG & GT & TT & $<0.0001$ \\
\hline & & & $1.56 \pm 0.47$ & $1.42 \pm 0.45$ & $1.29 \pm 0.40$ & \\
\hline \multirow[t]{2}{*}{ COL6A5 } & rs200982668 & $\mathrm{G} / \mathrm{A}$ & GG & GA & & $<0.0001$ \\
\hline & & & $1.53 \pm 0.47$ & $1.69 \pm 0.44$ & & \\
\hline \multirow[t]{4}{*}{ ZNF860 } & rs140232911 & $\mathrm{C} / \mathrm{T}$ & $\mathrm{CC}$ & $\mathrm{CT}$ & & 0.0003 \\
\hline & & & $1.54 \pm 0.47$ & $1.37 \pm 0.59$ & & \\
\hline & rs9261800 & $\mathrm{C} / \mathrm{G}$ & $\mathrm{CC}$ & CG & & $<0.0001$ \\
\hline & & & $1.54 \pm 0.47$ & $0.98 \pm 0.56$ & & \\
\hline \multirow[t]{2}{*}{$V P S 33 B$} & rs199921354 & $\mathrm{C} / \mathrm{T}$ & $\mathrm{CC}$ & $\mathrm{CT}$ & & $<0.0001$ \\
\hline & & & $1.53 \pm 0.47$ & $1.69 \pm 0.44$ & & \\
\hline \multirow[t]{2}{*}{$A D G R L 3$} & rs192210727 & $\mathrm{G} / \mathrm{T}$ & GG & GT & $\mathrm{TT}$ & $<0.0001$ \\
\hline & & & $1.53 \pm 0.47$ & $1.67 \pm 0.44$ & $1.91 \pm 0.49$ & \\
\hline \multirow[t]{2}{*}{ TMOD4 } & rs115287176 & $\mathrm{G} / \mathrm{A}$ & GG & GA & & $<0.0001$ \\
\hline & & & $1.53 \pm 0.47$ & $1.68 \pm 0.44$ & & \\
\hline \multirow[t]{2}{*}{ COL6A3 } & rs146092501 & $\mathrm{C} / \mathrm{T}$ & $\mathrm{CC}$ & $\mathrm{CT}$ & & $<0.0001$ \\
\hline & & & $1.53 \pm 0.47$ & $1.68 \pm 0.44$ & & \\
\hline \multirow[t]{2}{*}{ MARCHI } & rs61734696 & $\mathrm{G} / \mathrm{T}$ & GG & GT & & $<0.0001$ \\
\hline & & & $1.53 \pm 0.47$ & $1.69 \pm 0.44$ & & \\
\hline \multirow[t]{2}{*}{$P L C B 2$} & rs200787930 & $\mathrm{C} / \mathrm{T}$ & $\mathrm{CC}$ & $\mathrm{CT}$ & & $<0.0001$ \\
\hline & & & $1.53 \pm 0.47$ & $1.69 \pm 0.44$ & & \\
\hline \multirow[t]{2}{*}{ МOB3C } & rs139537100 & $\mathrm{C} / \mathrm{T}$ & $\mathrm{CC}$ & $\mathrm{CT}$ & & $<0.0001$ \\
\hline & & & $1.53 \pm 0.47$ & $1.68 \pm 0.44$ & & \\
\hline \multirow[t]{2}{*}{ CXCL8 } & rs188378669 & $\mathrm{G} / \mathrm{T}$ & GG & GT & & $<0.0001$ \\
\hline & & & $1.53 \pm 0.47$ & $1.69 \pm 0.44$ & & \\
\hline \multirow[t]{2}{*}{ EHD3 } & rs116417209 & $\mathrm{G} / \mathrm{A}$ & GG & GA & & $<0.0001$ \\
\hline & & & $1.54 \pm 0.47$ & $1.09 \pm 0.48$ & & \\
\hline \multirow[t]{2}{*}{ ZNF77 } & rs146879198 & $\mathrm{G} / \mathrm{A}$ & GG & GA & & $<0.0001$ \\
\hline & & & $1.53 \pm 0.47$ & $1.69 \pm 0.44$ & & \\
\hline \multirow[t]{2}{*}{ OR4F6 } & rs141569282 & $\mathrm{G} / \mathrm{A}$ & GG & GA & & $<0.0001$ \\
\hline & & & $1.48 \pm 0.46$ & $1.72 \pm 0.43$ & & \\
\hline \multirow[t]{2}{*}{ ACAD10 } & rs11066015 & $\mathrm{G} / \mathrm{A}$ & GG & GA & AA & $<0.0001$ \\
\hline & & & $1.57 \pm 0.48$ & $1.51 \pm 0.47$ & $1.43 \pm 0.43$ & \\
\hline CACNAID & rs35874056 & $\mathrm{G} / \mathrm{A}$ & GG & GA & & $<0.0001$ \\
\hline & & & $1.51 \pm 0.47$ & $0.94 \pm 0.64$ & & \\
\hline$A L D H 2$ & rs671 & $\mathrm{G} / \mathrm{A}$ & GG & GA & AA & $<0.0001$ \\
\hline & & & $1.57 \pm 0.48$ & $1.51 \pm 0.47$ & $1.43 \pm 0.43$ & \\
\hline & rs3764261 & $\mathrm{G} / \mathrm{T}$ & GG & GT & $\mathrm{TT}$ & $<0.0001$ \\
\hline & & & $1.50 \pm 0.46$ & $1.59 \pm 0.48$ & $1.72 \pm 0.49$ & \\
\hline & rs 247616 & $\mathrm{C} / \mathrm{T}$ & $\mathrm{CC}$ & $\mathrm{CT}$ & TT & $<0.0001$ \\
\hline & & & $1.50 \pm 0.46$ & $1.59 \pm 0.48$ & $1.71 \pm 0.50$ & \\
\hline$B R A P$ & rs3782886 & $\mathrm{A} / \mathrm{G}$ & $\mathrm{AA}$ & AG & GG & $<0.0001$ \\
\hline & & & $1.57 \pm 0.48$ & $1.52 \pm 0.47$ & $1.44 \pm 0.43$ & \\
\hline CETP & rs 1532624 & $\mathrm{G} / \mathrm{T}$ & GG & GT & $\mathrm{TT}$ & $<0.0001$ \\
\hline & & & $1.50 \pm 0.46$ & $1.56 \pm 0.47$ & $1.64 \pm 0.48$ & \\
\hline HECTD 4 & rs11066280 & $\mathrm{T} / \mathrm{A}$ & TT & TA & AA & $<0.0001$ \\
\hline & & & $1.57 \pm 0.47$ & $1.52 \pm 0.47$ & $1.44 \pm 0.42$ & \\
\hline HECTD4 & rs2074356 & $\mathrm{C} / \mathrm{T}$ & $\mathrm{CC}$ & $\mathrm{CT}$ & TT & $<0.0001$ \\
\hline & & & $1.57 \pm 0.48$ & $1.51 \pm 0.47$ & $1.44 \pm 0.43$ & \\
\hline LILRB2 & rs73055442 & $\mathrm{C} / \mathrm{T}$ & $\mathrm{CC}$ & $\mathrm{CT}$ & & $<0.0001$ \\
\hline & & & $1.54 \pm 0.47$ & $0.88 \pm 0.31$ & & \\
\hline
\end{tabular}


Table XI. Continued.

\begin{tabular}{|c|c|c|c|c|c|}
\hline \multirow{2}{*}{$\begin{array}{l}\text { Gene } \\
\text { DCLRE } 1 C\end{array}$} & \multicolumn{2}{|c|}{ SNP } & \multicolumn{2}{|c|}{ Serum HDL-cholesterol (mmol/l) } & \multirow{2}{*}{$\begin{array}{l}\text { P-value } \\
<0.0001\end{array}$} \\
\hline & rs 150854849 & $\mathrm{C} / \mathrm{T}$ & $\mathrm{CC}$ & $\mathrm{CT}$ & \\
\hline & & & $1.54 \pm 0.47$ & $0.92 \pm 0.68$ & \\
\hline \multirow[t]{2}{*}{$S P O P L$} & rs114501427 & $\mathrm{G} / \mathrm{A}$ & GG & GA & 0.0041 \\
\hline & & & $1.54 \pm 0.47$ & $1.41 \pm 0.58$ & \\
\hline
\end{tabular}

Data are presented as mean \pm standard deviation and were compared among genotypes by one-way analysis of variance. Based on Bonferroni's correction, $\mathrm{P}<0.0018$ (0.05/28) was considered statistically significant. SNP, single nucleotide polymorphism; HDL, high density lipoprotein.

Table XII. Association of SNPs identified in the present study to serum LDL-cholesterol concentration.

\begin{tabular}{|c|c|c|c|c|c|c|}
\hline \multirow{2}{*}{$\begin{array}{l}\text { Gene } \\
A P O E\end{array}$} & \multicolumn{2}{|c|}{ SNP } & \multicolumn{3}{|c|}{ Serum LDL-cholesterol (mmol/l) } & \multirow{2}{*}{$\begin{array}{l}\text { P-value } \\
<\mathbf{0 . 0 0 0 1}\end{array}$} \\
\hline & rs7412 & $\mathrm{C} / \mathrm{T}$ & $\mathrm{CC}$ & CT & $\mathrm{TT}$ & \\
\hline & & & $3.20 \pm 0.85$ & $2.74 \pm 0.80$ & $2.39 \pm 1.48$ & \\
\hline \multirow[t]{2}{*}{ COL6A3 } & rs 146092501 & $\mathrm{C} / \mathrm{T}$ & $\mathrm{CC}$ & $\mathrm{CT}$ & & 0.1168 \\
\hline & & & $3.16 \pm 0.86$ & $3.24 \pm 0.82$ & & \\
\hline \multirow[t]{2}{*}{ TMOD4 } & rs 115287176 & $\mathrm{G} / \mathrm{A}$ & GG & GA & & 0.1624 \\
\hline & & & $3.16 \pm 0.86$ & $3.23 \pm 0.82$ & & \\
\hline \multirow[t]{2}{*}{ CXCL8 } & rs 188378669 & $\mathrm{G} / \mathrm{T}$ & GG & GT & & 0.1158 \\
\hline & & & $3.16 \pm 0.86$ & $3.24 \pm 0.82$ & & \\
\hline \multirow[t]{2}{*}{$V P S 33 B$} & rs 199921354 & $\mathrm{C} / \mathrm{T}$ & $\mathrm{CC}$ & $\mathrm{CT}$ & & 0.1121 \\
\hline & & & $3.16 \pm 0.86$ & $3.24 \pm 0.81$ & & \\
\hline \multirow[t]{2}{*}{ ZNF77 } & rs 146879198 & $\mathrm{G} / \mathrm{A}$ & GG & GA & & 0.2277 \\
\hline & & & $3.16 \pm 0.86$ & $3.22 \pm 0.81$ & & \\
\hline \multirow[t]{2}{*}{$M O B 3 C$} & rs 139537100 & $\mathrm{C} / \mathrm{T}$ & $\mathrm{CC}$ & CT & & 0.2422 \\
\hline & & & $3.16 \pm 0.86$ & $3.21 \pm 0.82$ & & \\
\hline \multirow[t]{2}{*}{$P L C B 2$} & rs200787930 & $\mathrm{C} / \mathrm{T}$ & $\mathrm{CC}$ & $\mathrm{CT}$ & & 0.1860 \\
\hline & & & $3.16 \pm 0.86$ & $3.22 \pm 0.81$ & & \\
\hline \multirow[t]{2}{*}{$M U C 17$} & rs78010183 & $\mathrm{A} / \mathrm{T}$ & AA & AT & & 0.0096 \\
\hline & & & $3.15 \pm 0.87$ & $3.26 \pm 0.78$ & & \\
\hline \multirow[t]{2}{*}{$M A R C H I$} & rs61734696 & $\mathrm{G} / \mathrm{T}$ & GG & GT & & 0.3029 \\
\hline & & & $3.16 \pm 0.86$ & $3.21 \pm 0.80$ & & \\
\hline \multirow[t]{2}{*}{$A P O B$} & rs 13306206 & $\mathrm{G} / \mathrm{A}$ & GG & GA & AA & $<0.0001$ \\
\hline & & & $3.14 \pm 0.85$ & $3.42 \pm 0.98$ & $4.20 \pm 0.86$ & \\
\hline \multirow[t]{2}{*}{ COL6A5 } & rs200982668 & $\mathrm{G} / \mathrm{A}$ & GG & GA & & 0.3549 \\
\hline & & & $3.16 \pm 0.86$ & $3.20 \pm 0.80$ & & \\
\hline \multirow[t]{2}{*}{ РTCH2 } & rs 147284320 & $\mathrm{C} / \mathrm{T}$ & $\mathrm{CC}$ & $\mathrm{CT}$ & & 0.5075 \\
\hline & & & $3.17 \pm 0.82$ & $3.20 \pm 0.82$ & & \\
\hline \multirow[t]{2}{*}{$A D G R L 3$} & rs 192210727 & $\mathrm{G} / \mathrm{T}$ & GG & GT & $\mathrm{TT}$ & 0.6309 \\
\hline & & & $3.16 \pm 0.86$ & $3.20 \pm 0.81$ & $2.99 \pm 0.82$ & \\
\hline \multirow[t]{2}{*}{$A P O C 1$} & rs445925 & $\mathrm{C} / \mathrm{T}$ & $\mathrm{CC}$ & $\mathrm{CT}$ & $\mathrm{TT}$ & $<0.0001$ \\
\hline & & & $3.19 \pm 0.86$ & $2.96 \pm 0.86$ & $2.99 \pm 1.21$ & \\
\hline \multirow[t]{2}{*}{$H S P A 1 B$} & rs6457452 & $\mathrm{C} / \mathrm{T}$ & $\mathrm{CC}$ & $\mathrm{CT}$ & $\mathrm{TT}$ & $<0.0001$ \\
\hline & & & $3.14 \pm 0.85$ & $3.24 \pm 0.89$ & $3.38 \pm 0.88$ & \\
\hline \multirow[t]{4}{*}{ C6orf48 } & rs 11968400 & $\mathrm{C} / \mathrm{T}$ & $\mathrm{CC}$ & $\mathrm{CT}$ & $\mathrm{TT}$ & $<0.0001$ \\
\hline & & & $3.14 \pm 0.85$ & $3.24 \pm 0.89$ & $3.37 \pm 0.88$ & \\
\hline & rs 12210887 & $\mathrm{G} / \mathrm{T}$ & GG & GT & $\mathrm{TT}$ & $<0.0001$ \\
\hline & & & $3.14 \pm 0.85$ & $3.24 \pm 0.89$ & $3.37 \pm 0.89$ & \\
\hline \multirow[t]{2}{*}{$U B D$} & rs64036 & $\mathrm{C} / \mathrm{A}$ & $\mathrm{CC}$ & $\mathrm{CA}$ & $\mathrm{AA}$ & 0.0004 \\
\hline & & & $3.13 \pm 0.85$ & $3.19 \pm 0.87$ & $3.27 \pm 0.85$ & \\
\hline
\end{tabular}


Table XII. Continued.

\begin{tabular}{|c|c|c|c|c|c|c|}
\hline \multirow{2}{*}{$\frac{\text { Gene }}{C C H C R I}$} & \multicolumn{2}{|c|}{ SNP } & \multicolumn{3}{|c|}{ Serum LDL-cholesterol (mmol/l) } & \multirow{2}{*}{$\begin{array}{l}\text { P-value } \\
<0.0001\end{array}$} \\
\hline & rs147733073 & $\mathrm{C} / \mathrm{G}$ & $\mathrm{CC}$ & $\mathrm{CG}$ & GG & \\
\hline & & & $3.14 \pm 0.85$ & $3.23 \pm 0.89$ & $3.37 \pm 0.88$ & \\
\hline \multirow[t]{4}{*}{ VARS } & rs11751198 & $\mathrm{G} / \mathrm{A}$ & GG & GA & AA & $<0.0001$ \\
\hline & & & $3.14 \pm 0.85$ & $3.24 \pm 0.89$ & $3.37 \pm 0.89$ & \\
\hline & rs2596574 & G/A & GG & GA & AA & $<0.0001$ \\
\hline & & & $3.14 \pm 0.85$ & $3.24 \pm 0.89$ & $3.35 \pm 0.86$ & \\
\hline \multirow[t]{2}{*}{ LY6G6F } & rs9267547 & $\mathrm{G} / \mathrm{A}$ & GG & GA & $\mathrm{AA}$ & $<0.0001$ \\
\hline & & & $3.14 \pm 0.85$ & $3.23 \pm 0.89$ & $3.37 \pm 0.89$ & \\
\hline \multirow[t]{2}{*}{$N E U 1$} & rs13118 & T/A & TT & TA & AA & $<0.0001$ \\
\hline & & & $3.14 \pm 0.85$ & $3.24 \pm 0.89$ & $3.37 \pm 0.89$ & \\
\hline \multirow[t]{2}{*}{ SLC17A3 } & rs34902660 & $\mathrm{C} / \mathrm{A}$ & $\mathrm{CC}$ & $\mathrm{CA}$ & AA & $<0.0001$ \\
\hline & & & $3.14 \pm 0.85$ & $3.26 \pm 0.90$ & $3.35 \pm 0.91$ & \\
\hline \multirow[t]{2}{*}{ FAM65B } & rs150142878 & $\mathrm{C} / \mathrm{T}$ & $\mathrm{CC}$ & $\mathrm{CT}$ & $\mathrm{TT}$ & $<0.0001$ \\
\hline & & & $3.14 \pm 0.85$ & $3.30 \pm 0.94$ & $3.18 \pm 0.88$ & \\
\hline \multirow[t]{6}{*}{$T U B B$} & rs9500864 & $\mathrm{G} / \mathrm{A}$ & GG & GA & AA & 0.0002 \\
\hline & & & $3.13 \pm 0.85$ & $3.19 \pm 0.87$ & $3.28 \pm 0.90$ & \\
\hline & rs204999 & $\mathrm{A} / \mathrm{G}$ & AA & $\mathrm{AG}$ & GG & $<0.0001$ \\
\hline & & & $3.14 \pm 0.85$ & $3.24 \pm 0.89$ & $3.36 \pm 0.91$ & \\
\hline & rs3130663 & $\mathrm{A} / \mathrm{G}$ & AA & AG & GG & 0.0010 \\
\hline & & & $3.14 \pm 0.85$ & $3.22 \pm 0.88$ & $3.22 \pm 0.90$ & \\
\hline \multirow[t]{2}{*}{ VARS } & rs5030798 & $\mathrm{G} / \mathrm{A}$ & GG & GA & $\mathrm{AA}$ & $<0.0001$ \\
\hline & & & $3.14 \pm 0.85$ & $3.24 \pm 0.89$ & $3.37 \pm 0.89$ & \\
\hline \multirow[t]{2}{*}{$L Y 6 G 6 F$} & rs9267546 & G/A & GG & GA & AA & $<0.0001$ \\
\hline & & & $3.14 \pm 0.85$ & $3.24 \pm 0.89$ & $3.36 \pm 0.87$ & \\
\hline \multirow[t]{2}{*}{ PRRC $2 A$} & rs11538264 & $\mathrm{G} / \mathrm{A}$ & GG & GA & AA & $<0.0001$ \\
\hline & & & $3.14 \pm 0.85$ & $3.24 \pm 0.90$ & $3.36 \pm 0.88$ & \\
\hline \multirow[t]{2}{*}{$D P C R 1$} & rs11970154 & $\mathrm{G} / \mathrm{C}$ & GG & GC & $\mathrm{CC}$ & $<0.0001$ \\
\hline & & & $3.13 \pm 0.85$ & $3.23 \pm 0.89$ & $3.32 \pm 0.86$ & \\
\hline \multirow[t]{2}{*}{ MSH5 } & rs11754464 & $\mathrm{C} / \mathrm{T}$ & $\mathrm{CC}$ & $\mathrm{CT}$ & $\mathrm{TT}$ & $<0.0001$ \\
\hline & & & $3.14 \pm 0.85$ & $3.24 \pm 0.89$ & $3.37 \pm 0.89$ & \\
\hline \multirow[t]{2}{*}{ PCSK 9} & rs151193009 & $\mathrm{C} / \mathrm{T}$ & $\mathrm{CC}$ & $\mathrm{CT}$ & $\mathrm{TT}$ & $<0.0001$ \\
\hline & & & $3.17 \pm 0.86$ & $2.77 \pm 0.73$ & 2.05 & \\
\hline \multirow[t]{2}{*}{ GABBRI } & rs 29243 & $\mathrm{C} / \mathrm{T}$ & $\mathrm{CC}$ & CT & $\mathrm{TT}$ & 0.0001 \\
\hline & & & $3.14 \pm 0.85$ & $3.23 \pm 0.89$ & $3.34 \pm 0.86$ & \\
\hline \multirow[t]{2}{*}{ KIAA0319 } & rs 4576240 & $\mathrm{G} / \mathrm{T}$ & GG & GT & $\mathrm{TT}$ & $<0.0001$ \\
\hline & & & $3.14 \pm 0.85$ & $3.30 \pm 0.93$ & $3.17 \pm 0.89$ & \\
\hline \multirow[t]{2}{*}{ LY6G6F } & rs 17200983 & $\mathrm{C} / \mathrm{A}$ & $\mathrm{CC}$ & CA & AA & $<0.0001$ \\
\hline & & & $3.14 \pm 0.85$ & $3.24 \pm 0.89$ & $3.36 \pm 0.88$ & \\
\hline$T N X B$ & rs 140770834 & $\mathrm{C} / \mathrm{G}$ & $\mathrm{CC}$ & CG & GG & $<0.0001$ \\
\hline & & & $3.14 \pm 0.85$ & $3.24 \pm 0.89$ & $3.37 \pm 0.92$ & \\
\hline$T N X B$ & rs 11751545 & $\mathrm{~A} / \mathrm{C}$ & $\mathrm{AA}$ & $\mathrm{AC}$ & $\mathrm{CC}$ & $<0.0001$ \\
\hline & & & $3.14 \pm 0.85$ & $3.24 \pm 0.89$ & $3.37 \pm 0.92$ & \\
\hline DPCR1 & rs6933400 & $\mathrm{C} / \mathrm{T}$ & $\mathrm{CC}$ & $\mathrm{CT}$ & $\mathrm{TT}$ & $<0.0001$ \\
\hline & & & $3.13 \pm 0.85$ & $3.23 \pm 0.89$ & $3.32 \pm 0.87$ & \\
\hline$L Y 6 G 6 C$ & rs117894946 & $\mathrm{G} / \mathrm{C}$ & GG & $\mathrm{GC}$ & $\mathrm{CC}$ & $<0.0001$ \\
\hline & & & $3.14 \pm 0.85$ & $3.24 \pm 0.89$ & $3.36 \pm 0.88$ & \\
\hline SLC $44 A 4$ & rs117127493 & $\mathrm{G} / \mathrm{C}$ & GG & $\mathrm{GC}$ & $\mathrm{CC}$ & $<0.0001$ \\
\hline & & & $3.14 \pm 0.85$ & $3.24 \pm 0.89$ & $3.36 \pm 0.91$ & \\
\hline ZSCAN26 & rs76463649 & $\mathrm{A} / \mathrm{G}$ & AA & $\mathrm{AG}$ & GG & $<0.0001$ \\
\hline & & & $3.14 \pm 0.85$ & $3.24 \pm 0.89$ & $3.37 \pm 0.85$ & \\
\hline DHX16 & rs7749235 & $\mathrm{T} / \mathrm{C}$ & $\mathrm{TT}$ & $\mathrm{TC}$ & $\mathrm{CC}$ & 0.0010 \\
\hline & & & $3.14 \pm 0.85$ & $3.20 \pm 0.87$ & $3.28 \pm 0.90$ & \\
\hline
\end{tabular}


Table XII. Continued.

\begin{tabular}{|c|c|c|c|c|c|c|}
\hline \multirow{2}{*}{$\begin{array}{l}\text { Gene } \\
\text { LOC } 105375015\end{array}$} & \multicolumn{2}{|c|}{ SNP } & \multicolumn{3}{|c|}{ Serum LDL-cholesterol (mmol/l) } & \multirow{2}{*}{$\begin{array}{r}\text { P-value } \\
\mathbf{0 . 0 0 0 1}\end{array}$} \\
\hline & rs9264942 & $\mathrm{T} / \mathrm{C}$ & TT & $\mathrm{TC}$ & $\mathrm{CC}$ & \\
\hline & & & $3.13 \pm 0.84$ & $3.15 \pm 0.87$ & $3.25 \pm 0.88$ & \\
\hline \multirow[t]{2}{*}{ ZSCAN31 } & rs6922302 & $\mathrm{C} / \mathrm{G}$ & $\mathrm{CC}$ & $\mathrm{CG}$ & GG & $<0.0001$ \\
\hline & & & $3.14 \pm 0.85$ & $3.24 \pm 0.90$ & $3.37 \pm 0.85$ & \\
\hline \multirow[t]{2}{*}{$M D C 1$} & rs 2269702 & $\mathrm{~A} / \mathrm{G}$ & AA & $\mathrm{AG}$ & GG & 0.0013 \\
\hline & & & $3.14 \pm 0.85$ & $3.20 \pm 0.87$ & $3.28 \pm 0.91$ & \\
\hline \multirow[t]{2}{*}{$M D C 1$} & rs28986465 & $\mathrm{C} / \mathrm{T}$ & $\mathrm{CC}$ & $\mathrm{CT}$ & TT & 0.0013 \\
\hline & & & $3.14 \pm 0.85$ & $3.20 \pm 0.87$ & $3.28 \pm 0.91$ & \\
\hline \multirow[t]{2}{*}{$M D C 1$} & rs 2075015 & $\mathrm{G} / \mathrm{A}$ & GG & GA & AA & 0.0013 \\
\hline & & & $3.14 \pm 0.85$ & $3.20 \pm 0.87$ & $3.28 \pm 0.91$ & \\
\hline \multirow[t]{2}{*}{ DHX16 } & rs 2285321 & $\mathrm{~T} / \mathrm{C}$ & $\mathrm{TT}$ & $\mathrm{TC}$ & $\mathrm{CC}$ & 0.0011 \\
\hline & & & $3.14 \pm 0.85$ & $3.20 \pm 0.87$ & $3.28 \pm 0.91$ & \\
\hline \multirow[t]{2}{*}{$S K I V 2 L$} & rs492899 & $\mathrm{A} / \mathrm{G}$ & AA & $\mathrm{AG}$ & GG & 0.0001 \\
\hline & & & $3.14 \pm 0.85$ & $3.23 \pm 0.89$ & $3.36 \pm 0.91$ & \\
\hline \multirow[t]{4}{*}{$M D C 1$} & rs6924270 & $\mathrm{A} / \mathrm{G}$ & $\mathrm{AA}$ & $\mathrm{AG}$ & GG & 0.0012 \\
\hline & & & $3.14 \pm 0.85$ & $3.20 \pm 0.87$ & $3.28 \pm 0.91$ & \\
\hline & rs 2508015 & $\mathrm{C} / \mathrm{T}$ & $\mathrm{CC}$ & CT & $\mathrm{TT}$ & 0.0005 \\
\hline & & & $3.14 \pm 0.85$ & $3.22 \pm 0.88$ & $3.31 \pm 0.86$ & \\
\hline \multirow[t]{2}{*}{$T U B B$} & rs 25527 & $\mathrm{C} / \mathrm{T}$ & $\mathrm{CC}$ & CT & $\mathrm{TT}$ & 0.0008 \\
\hline & & & $3.14 \pm 0.86$ & $3.19 \pm 0.86$ & $3.27 \pm 0.89$ & \\
\hline \multirow[t]{2}{*}{ PPPIR18 } & rs9468805 & $\mathrm{G} / \mathrm{A}$ & GG & GA & AA & 0.0016 \\
\hline & & & $3.14 \pm 0.85$ & $3.20 \pm 0.87$ & $3.28 \pm 0.91$ & \\
\hline \multirow[t]{2}{*}{ DHX16 } & rs6937357 & $\mathrm{T} / \mathrm{C}$ & $\mathrm{TT}$ & $\mathrm{TC}$ & $\mathrm{CC}$ & 0.0016 \\
\hline & & & $3.14 \pm 0.85$ & $3.20 \pm 0.87$ & $3.28 \pm 0.91$ & \\
\hline \multirow[t]{2}{*}{ HECTD4 } & rs 11066280 & $\mathrm{~T} / \mathrm{A}$ & TT & TA & AA & 0.0005 \\
\hline & & & $3.12 \pm 0.88$ & $3.19 \pm 0.85$ & $3.24 \pm 0.81$ & \\
\hline \multirow[t]{2}{*}{ TRIM40 } & rs 2523995 & $\mathrm{G} / \mathrm{A}$ & GG & GA & AA & 0.0008 \\
\hline & & & $3.14 \pm 0.85$ & $3.22 \pm 0.89$ & $3.32 \pm 0.87$ & \\
\hline \multirow[t]{2}{*}{ PPPIRI8 } & rs6457254 & $\mathrm{C} / \mathrm{T}$ & $\mathrm{CC}$ & $\mathrm{CT}$ & $\mathrm{TT}$ & 0.0010 \\
\hline & & & $3.14 \pm 0.85$ & $3.20 \pm 0.87$ & $3.28 \pm 0.91$ & \\
\hline \multirow[t]{4}{*}{$T U B B$} & rs3132584 & $\mathrm{C} / \mathrm{A}$ & $\mathrm{CC}$ & $\mathrm{CA}$ & AA & 0.0008 \\
\hline & & & $3.13 \pm 0.86$ & $3.19 \pm 0.86$ & $3.27 \pm 0.90$ & \\
\hline & rs3130685 & $\mathrm{T} / \mathrm{C}$ & TT & $\mathrm{TC}$ & $\mathrm{CC}$ & 0.0234 \\
\hline & & & $3.20 \pm 0.89$ & $3.14 \pm 0.84$ & $3.14 \pm 0.85$ & \\
\hline \multirow[t]{2}{*}{ GIT2 } & rs2292354 & $\mathrm{C} / \mathrm{T}$ & $\mathrm{CC}$ & $\mathrm{CT}$ & $\mathrm{TT}$ & 0.0077 \\
\hline & & & $3.18 \pm 0.86$ & $3.15 \pm 0.87$ & $3.05 \pm 0.82$ & \\
\hline \multirow[t]{4}{*}{ PPP1R18 } & rs2394392 & $\mathrm{T} / \mathrm{G}$ & $\mathrm{TT}$ & $\mathrm{TG}$ & GG & 0.0013 \\
\hline & & & $3.14 \pm 0.85$ & $3.20 \pm 0.87$ & $3.28 \pm 0.91$ & \\
\hline & rs 2524272 & $\mathrm{~T} / \mathrm{C}$ & TT & $\mathrm{TC}$ & $\mathrm{CC}$ & 0.0005 \\
\hline & & & $3.13 \pm 0.86$ & $3.18 \pm 0.87$ & $3.27 \pm 0.87$ & \\
\hline
\end{tabular}

Data are presented as mean \pm standard deviation and were compared among genotypes by one-way analysis of variance. Based on Bonferroni's correction, $\mathrm{P}<0.0008(0.05 / 65)$ was considered statistically significant (indicated in bold). SNP, single nucleotide polymorphism; LDL, low density lipoprotein.

were associated with diabetes mellitus-associated phenotypes. The remaining 16 genes (PTCH2, MOB3C, TMOD4, COL6A3, COL6A5, ADGRL3, KIAA0319, FAM65B, ZSCAN26, UBD, DHX16, LOC105375015, NEU1, MUC17, GIT2, ZNF77) had not been related to circulating LDL-cholesterol or other dyslipidemia-related phenotypes.
Network analysis of genes identified in the present study. Network analysis of the 10 genes (MOB3C, TMOD4, LPGAT1, EHD3, COL6A3, ZNF860, CACNA1D, COL6A5, DCLRE1C, ZNF77) or three genes (KIAA0319, FAM65B, UBD) associated in the present study with hypo-HDL-cholesterolemia or hyper-LDL-cholesterolemia, respectively, was performed 


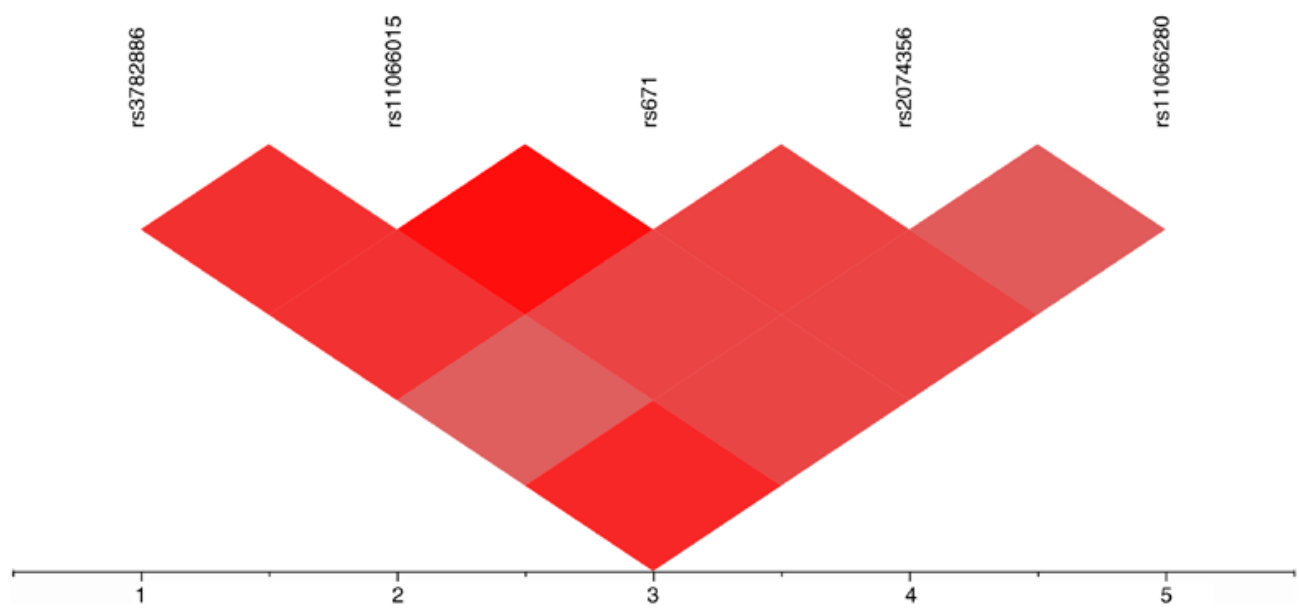

Figure 3. LD map for five SNPs at chromosomal region 12q24.12 to 12q24.13 associated with hypo-HDL-cholesterolemia. LD was calculated as the square of the correlation coefficient $\left(r^{2}\right)$, with the extent of LD increasing according to the color order of gray to red. LD, linkage disequilibrium; SNP, single nucleotide polymorphism.

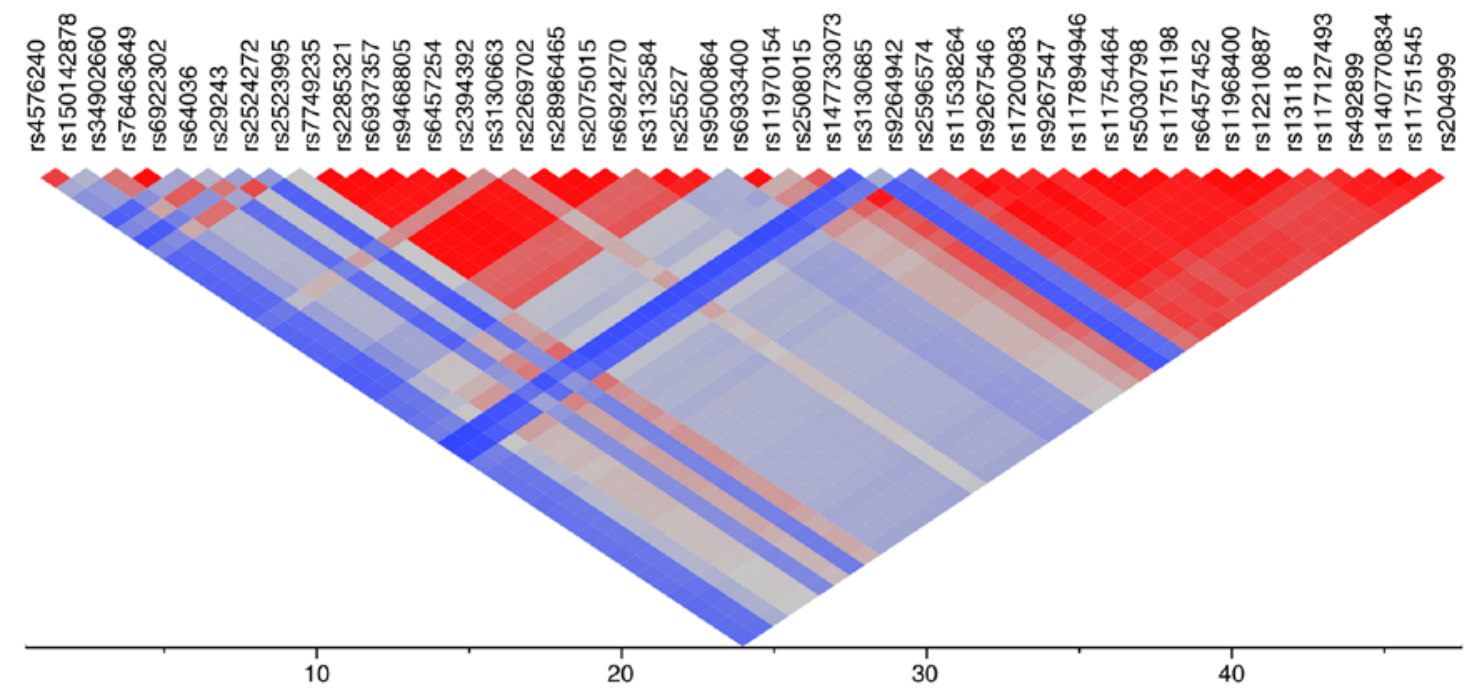

Figure 4. LD map for 47 SNPs at 6p22.3 to 6p21.3 associated with hyper-LDL-cholesterolemia. LD was calculated as the square of the correlation coefficient $\left(r^{2}\right)$, with the extent of LD increasing according to the color order of blue to gray to red. LD, linkage disequilibrium; SNP, single nucleotide polymorphism.

to predict functional gene-gene interactions, with the use of the GeneMANIA Cytoscape plugin (http://apps.cytoscape. org/apps/genemania; Donnelly Centre for Cellular and Biomolecular Research, University of Toronto, Toronto, Canada) (35-37) and Cytoscape v3.4.0 software (http://www.cytoscape.org; The Cytoscape Consortium, San Diego, CA, USA) (38). Given that LOC105375015 protein has not been characterized, it was not examined. A set of 50 dyslipidemia-related genes were selected from the DisGeNET database (http://www.disgenet.org/web/DisGeNET; Integrative Biomedical Informatics Group, Research Programme on Biomedical Informatics, Barcelona Biomedical Research Park, Barcelona, Spain) $(39,40)$ according to the rank order (high to low) of scores for association with dyslipidemia. The network analysis revealed that the 10 or three genes associated in the present study with hypo-HDL-cholesterolemia or hyper-LDL-cholesterolemia, respectively, have potential direct or indirect interactions with the 50 genes previously demonstrated to be associated with dyslipidemia (Fig. 5).

\section{Discussion}

Dyslipidemia is an important public health problem because of its high prevalence and role as a risk factor for more serious conditions, including coronary artery disease, ischemic stroke, and colorectal cancer (9-12). The identification of genetic variants that confer susceptibility to dyslipidemia is thus clinically important for prevention of these conditions. The present study has performed novel EWASs for hypertriglyceridemia, hypo-HDL-cholesterolemia, and hyper-LDL-cholesterolemia in subjects with early-onset forms of these disorders, which may have a greater genetic component compared with late-onset forms.

Among 25 SNPs in six genes and five chromosomal loci significantly associated with early-onset hypertriglyceridemia, the present study newly identified rs11085421 at 19p12 as a susceptibility locus for this condition. This SNP was significantly associated with serum triglyceride concentration, with the minor $C$ allele being protective against hypertriglyceridemia. 


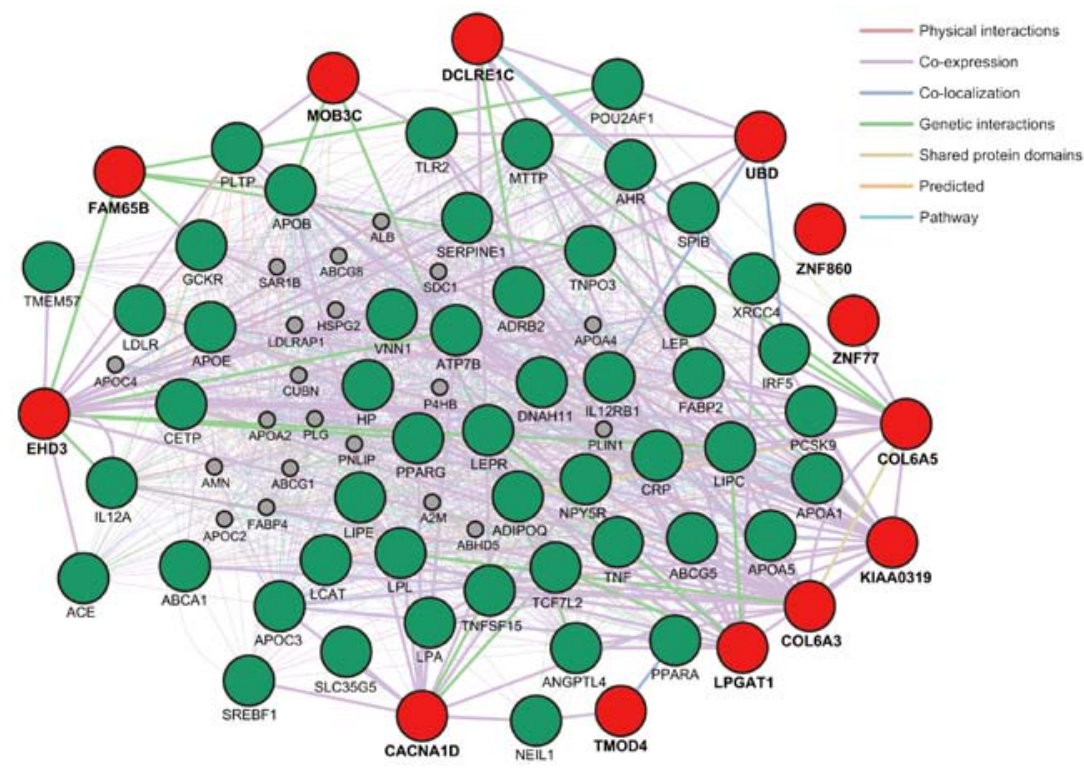

Figure 5. Network analysis of the genes identified in the present study with known dyslipidemia-related genes. The 10 genes (MOB3C, TMOD4, LPGAT1, EHD3, COL6A3, ZNF860, CACNA1D, COL6A5, DCLRE1C, ZNF77) and three genes (KIAA0319, FAM65B, UBD) associated in the present study with hypo-HDL-cholesterolemia and hyper-LDL-cholesterolemia, respectively, are indicated in red circles. The 50 dyslipidemia-related genes selected from the DisGeNET database (http://www.disgenet.org/web/ DisGeNET) are indicated in green circles. Interactions between red circles or between red and green circles are indicated by bold lines. Molecules represented by gray circles are putative mediators of interactions between the genes.

Among 28 SNPs in 24 genes and two chromosomal loci significantly associated with hypo-HDL-cholesterolemia, the current study newly identified 13 genes $(M O B 3 C, T M O D 4$ LPGAT1, EHD3, SPOPL, COL6A3, ZNF860, CACNA1D, COL6A5, ADGRL3, DCLRE1C, OR4F6, ZNF77) as potential susceptibility loci for this condition. SNPs of 12 of these genes were significantly associated with serum HDL-cholesterol levels, with minor alleles of LPGAT1, EHD3, ZNF860, CACNA1D, and DCLREIC representing risk factors for hypo-HDL-cholesterolemia and minor alleles of MOB3C, TMOD4, COL6A3, COL6A5, ADGRL3, OR4F6, and $Z N F 77$ being protective against this condition. Given that rs114501427 of SPOPL was not significantly related to serum HDL-cholesterol concentration, this gene was excluded as a novel susceptibility locus. Furthermore, given that significant LD was apparent among SNPs of $A D G R L 3$, $C X C L 8$, and MARCH1, as well as between SNPs of OR4F6 and $V P S 33 B, A D G R L 3$ and $O R 4 F 6$ were also removed from the final list of novel significant loci. In addition, significant LD was detected between SNPs of MOB3C and TMOD4 as well as between those of ZNF860 and CACNA1D. We thus identified eight novel loci (MOB3C-TMOD4, LPGAT1, EHD3, COL6A3, ZNF860-CACNA1D, COL6A5, DCLRE1C, ZNF77) that confer susceptibility to early-onset hypo-HDL-cholesterolemia.

Among 65 SNPs in 44 genes and two chromosomal loci significantly associated with hyper-LDL-cholesterolemia, the present study newly identified 16 genes (PTCH2, MOB3C, TMOD4, COL6A3, COL6A5, ADGRL3, KIAA0319, FAM65B, ZSCAN26, UBD, DHX16, LOC105375015, NEU1, MUC17, GIT2, ZNF77) as potential susceptibility loci for this condition. SNPs of six of these genes (KIAA0319, FAM65B, ZSCAN26, UBD, LOC105375015, NEU1) were significantly associated with serum LDL-cholesterol levels, with minor alleles of these genes representing risk factors for
hyper-LDL-cholesterolemia. Given that SNPs of 10 genes (PTCH2, MOB3C, TMOD4, COL6A3, COL6A5, ADGRL3, DHX16, MUC17, GIT2, ZNF77) were not significantly related to the serum LDL-cholesterol concentration, these genes were removed from the list of novel susceptibility loci, even though this discrepancy may be attributable to the effects of lipid-lowering medication. Given that rs13118 of NEU1 was in significant LD with rs492899 of SKIV2L in a large LD block and that rs76463649 of ZSCAN26 was in LD with SNPs of SLC17A3, ZSCAN31, GABBR1, and TRIM40, both NEU1 and ZSCAN26 were also excluded from the final results. In addition, significant LD was detected between SNPs of KIAA0319 and FAM65B. We thus identified three novel loci (KIAA0319-FAM65B, UBD, $L O C 105375015)$ that confer susceptibility to early-onset hyper-LDL-cholesterolemia.

A recent study has reported that network analysis of functional gene-gene interactions may be informative with regard both to clarification of biological processes underlying coronary artery disease and to the identification of therapeutic targets for this condition (41). A network analysis was therefore performed in the present study in order to predict biological processes related to the identified genes and the interactions of these genes with those previously known to be associated with dyslipidemia. The results from the network analysis revealed that the 10 or three genes associated in the present study with hypo-HDL-cholesterolemia or hyper-LDL-cholesterolemia respectively, had direct or indirect interactions with the 50 dyslipidemia-related genes selected from the DisGeNET database $(39,40)$. The underlying molecular mechanisms of these interactions, however, remain to be elucidated.

Our group has previously reported that two, three and nine SNPs were associated with hypertriglyceridemia $\left(\mathrm{P}<1.71 \times 10^{-4}\right)$, hypo-HDL-cholesterolemia $\left(\mathrm{P}<1.44 \times 10^{-4}\right)$, 





Table XIV. Relation of genes, chromosomal loci and SNPs associated with hypo-HDL-cholesterolemia in the present study to previously examined dyslipidemia-related phenotypes.

\begin{tabular}{|c|c|c|c|c|}
\hline Gene/chr locus & SNP & $\mathrm{Chr}$ & Position & Previously examined phenotypes \\
\hline MOB3C & rs 139537100 & 1 & 46615006 & None \\
\hline TMOD4 & rs 115287176 & 1 & 151170961 & None \\
\hline$L P G A T 1$ & rs 150552771 & 1 & 211783358 & None \\
\hline EHD3 & rs 116417209 & 2 & 31249417 & None \\
\hline$S P O P L$ & rs 114501427 & 2 & 138568946 & None \\
\hline COL6A3 & rs 146092501 & 2 & 237371861 & None \\
\hline ZNF860 & rs 140232911 & 3 & 31989561 & None \\
\hline CACNAID & rs35874056 & 3 & 53702798 & None \\
\hline COL6A5 & rs200982668 & 3 & 130470894 & None \\
\hline$A D G R L 3$ & rs 192210727 & 4 & 61909615 & None \\
\hline CXCL8 & rs 188378669 & 4 & 73741568 & Total cholesterol (23063622) \\
\hline$M A R C H 1$ & rs61734696 & 4 & 164197303 & Adiponectin concentrations (20887962) \\
\hline $6 \mathrm{p} 22.1$ & rs9261800 & 6 & 30408822 & Type 1 diabetes (17554300) \\
\hline DCLRE1C & rs 150854849 & 10 & 14934704 & None \\
\hline APOA5 & rs2075291 & 11 & 116790676 & $\begin{array}{l}\text { HDL-cholesterol }(23063622,20686565,22629316, \\
21386085)\end{array}$ \\
\hline$B R A P$ & rs3782886 & 12 & 111672685 & HDL-cholesterol (21572416) \\
\hline$A C A D 10$ & rs 11066015 & 12 & 111730205 & LDL-cholesterol (20686565) \\
\hline$A L D H 2$ & rs671 & 12 & 111803962 & HDL-cholesterol (21572416) \\
\hline \multirow[t]{2}{*}{ HECTD4 } & rs2074356 & 12 & 112207597 & $\begin{array}{l}\text { HDL-cholesterol }(21572416,21909109, \\
22751097)\end{array}$ \\
\hline & rs 11066280 & 12 & 112379979 & \\
\hline$P L C B 2$ & rs200787930 & 15 & 40289298 & Triglycerides (23063622) \\
\hline$V P S 33 B$ & rs 199921354 & 15 & 91013841 & Type 2 diabetes (22885922) \\
\hline OR4F6 & rs 141569282 & 15 & 101806068 & None \\
\hline $16 q 13$ & rs247616 & 16 & 56955678 & $\begin{array}{l}\text { HDL-cholesterol }(20686565,19060906,20339536, \\
20838585,19060911,20031538)\end{array}$ \\
\hline $16 q 13$ & rs3764261 & 16 & 56959412 & $\begin{array}{l}\text { HDL-cholesterol }(20686565,23063622,19060911, \\
21943158, \quad 21347282, \quad 18193043,20694148, \\
21589926,19802338,19913121)\end{array}$ \\
\hline CETP & rs 1532624 & 16 & 56971567 & $\begin{array}{l}\text { HDL-cholesterol }(20686565,23063622,22629316, \\
21347282,21589926,19060911,20031564, \\
19060906,18193044,21943158)\end{array}$ \\
\hline ZNF77 & rs 146879198 & 19 & 2934109 & None \\
\hline LILRB2 & rs73055442 & 19 & 54279838 & HDL-cholesterol (20686565) \\
\hline
\end{tabular}

Data were obtained from the Genome-Wide Repository of Associations Between SNPs and Phenotypes (GRASP) Search database (https://grasp. nhlbi.nih.gov/Search.aspx) with a P-value of $<1.0 \times 10^{-6}$. Numbers in parentheses are PubMed IDs. SNP, single nucleotide polymorphism; Chr, chromosome.

and hyper-LDL-cholesterolemia $\left(\mathrm{P}<1.10 \times 10^{-4}\right)$, respectively, as determined by multivariable logistic regression analysis with adjustment for age and sex, following an initial EWAS screening of allele frequencies among subjects with early- or late-onset forms of these conditions (28). The associations of the two SNPs [rs10790162 $\left(\mathrm{P}=3.58 \times 10^{-24}\right)$ and $\mathrm{rs} 7350481$ $\left.\left(\mathrm{P}=7.94 \times 10^{-23}\right)\right]$ with hypertriglyceridemia were replicated $(\mathrm{P}<0.05)$ in the present study. The associations of two of the three SNPs [rs147317864 $(\mathrm{P}=0.0139)$ and $\mathrm{rs} 12229654$ $(\mathrm{P}=0.0001)]$ with hypo-HDL-cholesterolemia were replicated in the present study. The associations of eight of the nine
SNPs [rs7771335 $\left(\mathrm{P}=1.00 \times 10^{-7}\right), \mathrm{rs} 2071653\left(\mathrm{P}=1.61 \times 10^{-5}\right)$, rs2853969 $\left(\mathrm{P}=6.16 \times 10^{-8}\right), \operatorname{rs} 2269704\left(\mathrm{P}=2.92 \times 10^{-7}\right)$, rs2269703 $\left(\mathrm{P}=3.67 \times 10^{-7}\right), \quad$ rs $495089(\mathrm{P}=0.0001)$, rs2269702 $\left(\mathrm{P}=5.13 \times 10^{-7}\right)$ and $\left.\operatorname{rs} 1233399(\mathrm{P}=0.0009)\right]$ with hyper-LDL-cholesterolemia were replicated in the present study. Although the association of most SNPs with dyslipidemia identified in our previous study (28) was replicated, genetic variants associated with hypertriglyceridemia, hypo-HDL-cholesterolemia, or hyper-LDL-cholesterolemia appear to differ, at least in part, between early- and late-onset forms of the diseases. 
Table XV. Relation of genes, chromosomal loci and SNPs associated with hyper-LDL-cholesterolemia in the present study to previously examined dyslipidemia-related phenotypes.

\begin{tabular}{|c|c|c|c|c|}
\hline Gene/chr locus & SNP & $\mathrm{Chr}$ & Position & Previously examined phenotypes \\
\hline PTCH2 & rs147284320 & 1 & 44828589 & None \\
\hline$M O B 3 C$ & rs139537100 & 1 & 46615006 & None \\
\hline PCSK9 & rs151193009 & 1 & 55043912 & $\begin{array}{l}\text { LDL-cholesterol (23063622, 21347282, } 18193044, \\
20686565,22629316)\end{array}$ \\
\hline TMOD4 & rs115287176 & 1 & 151170961 & None \\
\hline$A P O B$ & rs13306206 & 2 & 21019859 & LDL-cholesterol (20686565, 23202125, 23063622) \\
\hline COL6A3 & rs146092501 & 2 & 237371861 & None \\
\hline COL6A5 & rs200982668 & 3 & 130470894 & None \\
\hline$A D G R L 3$ & rs192210727 & 4 & 61909615 & None \\
\hline CXCL8 & rs188378669 & 4 & 73741568 & Total cholesterol (23063622) \\
\hline$M A R C H 1$ & rs61734696 & 4 & 164197303 & Adiponectin concentrations (20887962) \\
\hline KIAA0319 & rs4576240 & 6 & 24596250 & None \\
\hline FAM65B & rs150142878 & 6 & 24847657 & None \\
\hline SLC17A3 & rs34902660 & 6 & 25850874 & Serum urate (23263486), type 1 diabetes (17554300) \\
\hline ZSCAN26 & rs76463649 & 6 & 28271963 & None \\
\hline ZSCAN31 & rs6922302 & 6 & 28327533 & Type 1 diabetes (17554300) \\
\hline$U B D$ & rs64036 & 6 & 29559490 & None \\
\hline$G A B B R 1$ & rs29243 & 6 & 29631325 & Type 1 diabetes (17554300) \\
\hline $6 \mathrm{p} 22.1$ & rs2524272 & 6 & 29714623 & Type 1 diabetes (17554300) \\
\hline TRIM40 & rs2523995 & 6 & 30134407 & Type 1 diabetes $(17554300,17632545)$ \\
\hline \multirow[t]{3}{*}{ DHX16 } & rs7749235 & 6 & 30667816 & None \\
\hline & rs2285321 & 6 & 30670221 & \\
\hline & rs6937357 & 6 & 30672547 & \\
\hline \multirow{3}{*}{ PPPIR18 } & rs9468805 & 6 & 30675932 & Type 1 diabetes (17632545) \\
\hline & rs6457254 & 6 & 30681357 & \\
\hline & rs2394392 & 6 & 30682541 & \\
\hline $6 \mathrm{p} 21.3$ & rs3130663 & 6 & 30698817 & Type 1 diabetes $(17632545,17554300)$ \\
\hline \multirow[t]{4}{*}{$M D C 1$} & rs2269702 & 6 & 30707358 & $\begin{array}{l}\text { Fat mass (19584900), type } 1 \text { diabetes (17554300), body } \\
\text { mass index (19584900) }\end{array}$ \\
\hline & rs28986465 & 6 & 30712785 & \\
\hline & rs2075015 & 6 & 30712831 & \\
\hline & rs6924270 & 6 & 30714203 & \\
\hline \multirow[t]{3}{*}{$T U B B$} & rs3132584 & 6 & 30720650 & Type 1 diabetes (17554300) \\
\hline & rs25527 & 6 & 30723161 & \\
\hline & rs9500864 & 6 & 30725455 & \\
\hline \multirow[t]{2}{*}{$D P C R l$} & rs6933400 & 6 & 30939399 & Type 1 diabetes $(17554300,17632545)$ \\
\hline & rs11970154 & 6 & 30952101 & \\
\hline $6 \mathrm{p} 21.3$ & rs2508015 & 6 & 31042423 & Type 1 diabetes (17632545), triglycerides (20686565) \\
\hline CCHCRl & rs147733073 & 6 & 31145462 & Triglycerides (20686565) \\
\hline $6 \mathrm{p} 21.3$ & rs3130685 & 6 & 31238429 & Triglycerides (20686565), type 1 diabetes (17554300) \\
\hline LOC105375015 & rs9264942 & 6 & 31306603 & None \\
\hline $6 \mathrm{p} 21.3$ & rs2596574 & 6 & 31366397 & $\begin{array}{l}\text { LDL-cholesterol (23063622), total cholesterol } \\
\text { (23063622), type } 1 \text { diabetes }(17554300,17632545)\end{array}$ \\
\hline$P R R C 2 A$ & rs11538264 & 6 & 31635412 & $\begin{array}{l}\text { Type } 1 \text { diabetes }(17554300,17632545), \text { triglycerides } \\
(20686565) \text {, total cholesterol }(20686565)\end{array}$ \\
\hline \multirow[t]{3}{*}{$L Y 6 G 6 F$} & rs9267546 & 6 & 31705659 & Triglycerides (20686565) \\
\hline & rs17200983 & 6 & 31707506 & \\
\hline & rs9267547 & 6 & 31707724 & \\
\hline$L Y 6 G 6 C$ & rs117894946 & 6 & 31719250 & Type 1 diabetes $(17554300,17632545)$ \\
\hline MSH5 & rs11754464 & 6 & 31755958 & $\begin{array}{l}\text { Type } 1 \text { diabetes }(17554300,17632545), \text { triglycerides } \\
(20686565) \text {, total cholesterol }(20686565)\end{array}$ \\
\hline
\end{tabular}


Table XV. Continued.

\begin{tabular}{|c|c|c|c|c|}
\hline Gene/chr locus & SNP & Chr & Position & Previously examined phenotypes \\
\hline \multirow[t]{2}{*}{$V A R S$} & rs5030798 & 6 & 31779733 & Type 1 diabetes (17632545) \\
\hline & rs11751198 & 6 & 31785749 & \\
\hline$H S P A I B$ & rs6457452 & 6 & 31827773 & Type 1 diabetes $(17554300,17632545)$ \\
\hline C6orf48 & rs11968400 & 6 & 31836952 & $\begin{array}{l}\text { Triglycerides (20686565), total cholesterol (20686565), } \\
\text { type } 1 \text { diabetes }(17632545)\end{array}$ \\
\hline $6 \mathrm{p} 21.3$ & rs12210887 & 6 & 31847946 & $\begin{array}{l}\text { Type } 1 \text { diabetes }(17554300,17632545,) \text {, triglycerides } \\
\text { (20686565), total cholesterol ( } 20686565)\end{array}$ \\
\hline$N E U 1$ & rs13118 & 6 & 31859509 & None \\
\hline SLC44A4 & rs117127493 & 6 & 31869232 & $\begin{array}{l}\text { Type } 1 \text { diabetes }(17632545) \text {, triglycerides }(20686565) \text {, } \\
\text { total cholesterol }(20686565)\end{array}$ \\
\hline$S K I V 2 L$ & rs492899 & 6 & 31965741 & $\begin{array}{l}\text { LDL-cholesterol }(20686565), \text { type } 1 \text { diabetes } \\
(17554300,17632545), \text { triglycerides }(20686565), \text { total } \\
\text { cholesterol }(20686565)\end{array}$ \\
\hline \multirow[t]{2}{*}{$T N X B$} & rs140770834 & 6 & 32064851 & $\begin{array}{l}\text { Type } 1 \text { diabetes }(17554300,17632545) \text {, triglycerides } \\
(20686565) \text {, total cholesterol }(20686565)\end{array}$ \\
\hline & rs11751545 & 6 & 32073266 & \\
\hline $6 \mathrm{p} 21.3$ & rs204999 & 6 & 32142202 & Type 1 diabetes (17632545) \\
\hline MUC17 & rs78010183 & 7 & 101035329 & None \\
\hline GIT2 & rs2292354 & 12 & 109930396 & None \\
\hline HECTD4 & rs11066280 & 12 & 112379979 & $\begin{array}{l}\text { LDL-cholesterol }(21572416,20686565) \text {, } \\
\text { HDL-cholesterol }(21572416,21909109,22751097)\end{array}$ \\
\hline PLCB2 & rs200787930 & 15 & 40289298 & Triglycerides (23063622) \\
\hline$V P S 33 B$ & rs199921354 & 15 & 91013841 & Type 2 diabetes (22885922) \\
\hline ZNF77 & rs146879198 & 19 & 2934109 & None \\
\hline$A P O E$ & rs7412 & 19 & 44908822 & $\begin{array}{l}\text { LDL-cholesterol }(23100282,23063622,20686565, \\
22629316,19060911)\end{array}$ \\
\hline$A P O C 1$ & rs445925 & 19 & 44912383 & $\begin{array}{l}\text { LDL-cholesterol }(20686565,21347282,18193044, \\
23063622,18193043,20864672,23100282)\end{array}$ \\
\hline
\end{tabular}

Data were obtained from the Genome-Wide Repository of Associations Between SNPs and Phenotypes (GRASP) Search database (https://grasp.nhlbi.nih.gov/Search.aspx) with a P-value of $<1.0 \times 10^{-6}$. Numbers in parentheses are PubMed IDs. SNP, single nucleotide polymorphism; Chr, chromosome.

There are several limitations to the present study. Firstly, given that the results were not replicated, their validation will be necessary in independent study populations or in other ethnic groups. The identified SNPs were not tested in a clinical setting to validate the assessment of genetic risk for dyslipidemia in patients. In addition, it is possible that SNPs identified in the present study may be in LD with other genetic variants in the same gene or in nearby genes that are actually responsible for the development of hypertriglyceridemia, hypo-HDL-cholesterolemia, or hyper-LDL-cholesterolemia. Finally, the functional relevance of identified SNPs to the pathogenesis of hypertriglyceridemia, hypo-HDL-cholesterolemia, or hyper-LDL-cholesterolemia remains to be elucidated.

In conclusion, the present study has identified chromosome 19p12, eight loci (MOB3C-TMOD4, LPGAT1, EHD3, COL6A3, ZNF860-CACNA1D, COL6A5, DCLRE1C, ZNF77) and three loci (KIAA0319-FAM65B, UBD, LOC105375015) that confer susceptibility to early-onset hypertriglyceridemia, hypo-HDL-cholesterolemia and hyper-LDL-cholesterolemia, respectively. Determination of genotypes for the SNPs at these loci may prove informative for assessment of genetic risk for hypertriglyceridemia, hypo-HDL-cholesterolemia and hyper-LDL-cholesterolemia in the Japanese population.

\section{Acknowledgments}

Not applicable.

\section{Funding}

This work was supported by CREST, Japan Science and Technology Agency (grant no. JPMJCR1302; to YYam, JS and IT).

\section{Availability of data and materials}

All data underlying the findings described in the article are available on request from the corresponding author.

\section{Authors' contributions}

YYam contributed to conception and design of the study; to acquisition, analysis, and interpretation of the data; and to drafting of the manuscript. $\mathrm{KK}, \mathrm{MO}, \mathrm{HH}$, and TF each 
contributed to acquisition of the data and to revision of the manuscript. YYas, IT, and JS contributed to analysis and interpretation of the data as well as to revision of the manuscript. All authors read and approved the final manuscript.

\section{Ethics approval and consent to participate}

The study protocol complied with the Declaration of Helsinki and was approved by the Committees on the Ethics of Human Research of Mie University Graduate School of Medicine, Hirosaki University Graduate School of Medicine, and participating hospitals (Gifu Prefectural Tajimi Hospital, Gifu Prefectural General Medical Center, Japanese Red Cross Nagoya First Hospital, Northern Mie Medical Center Inabe General Hospital, and Hirosaki Stroke and Rehabilitation Center). Written informed consent for participation in the study was obtained from all subjects.

\section{Patient consent for publication}

Not applicable.

\section{Competing interests}

The authors declare that they have no competing interests.

\section{References}

1. Durrington P: Dyslipidaemia. Lancet 362: 717-731, 2003.

2. Paththinige CS, Sirisena ND and Dissanayake V: Genetic determinants of inherited susceptibility to hypercholesterolemia-a comprehensive literature review. Lipids Health Dis 16: 103, 2017.

3. Dron JS and Hegele RA: Genetics of triglycerides and the risk of atherosclerosis. Curr Atheroscler Rep 19: 31, 2017.

4. Soutar AK and Naoumova RP: Mechanism of disease: Genetic causes of familial hypercholesterolemia. Nat Clin Pract Cardiovasc Med 4: 214-225, 2007.

5. Heller DA, DeFaire U, Pedersen N, Dahlén G and McClearn GE: Genetic and environmental influences on serum lipid levels in twins. N Engl J Med 328: 1150-1156, 1993.

6. Abney M, McPeek MS and Ober C: Broad and narrow heritabilities of quantitative traits in a founder population. Am J Hum Genet 68: 1302-1307, 2001.

7. van Dongen J, Willemsen G, Chen WM, de Geus EJ and Boomsma DI: Heritability of metabolic syndrome traits in a large population-based sample. J Lipid Res 54: 2914-2923, 2013.

8. Woo JG, Morrison JA, Stroop DM, Aronson Friedman L and Martin LJ: Genetic architecture of lipid traits changes over time and differs by race: Princeton Lipid Follow-up Study. J Lipid Res 55: 1515-1524, 2014.

9. Benjamin EJ, Virani SS, Callaway $\mathrm{CW}$, Chamberlain AM, Chang AR, Cheng S, Chiuve SE, Cushman M, Delling FN, Deo R, et al: Heart disease and stroke statistics-2018 update: A report from the American Heart Association. Circulation 137: e67-e492, 2018.

10. Law MR, Wald NJ and Rudnicka AR: Quantifying effect of statins on low density lipoprotein cholesterol, ischaemic heart disease, and stroke: Systematic review and meta-analysis. $\mathrm{Br}$ Med J 326: 1423, 2003.

11. Agnoli C, Grioni S, Sieri S, Sacerdote C, Vineis P, Tumino R Giurdanella MC, Pala V, Mattiello A, Chiodini P, et al: Colorectal cancer risk and dyslipidemia: A case-cohort study nested in an Italian multicentre cohort. Cancer Epidemiol 38: 144-151, 2014.

12. Yao X and Tian Z: Dyslipidemia and colorectal cancer risk: A meta-analysis of prospective studies. Cancer Causes Control 26: 257-268, 2015.

13. Kathiresan S, Melander O, Guiducci C, Surti A, Burtt NP, Rieder MJ, Cooper GM, Roos C, Voight BF, Havulinna AS, et al: Six new loci associated with blood low-density lipoprotein cholesterol, high-density lipoprotein cholesterol or triglycerides in humans. Nat Genet 40: 189-197, 2008.
14. Kathiresan S, Willer CJ, Peloso GM, Demissie S, Musunuru K, Schadt EE, Kaplan L, Bennett D, Li Y, Tanaka T, et al: Common variants at 30 loci contribute to polygenic dyslipidemia. Nat Genet 41: 56-65, 2009

15. Aulchenko YS, Ripatti S, Lindqvist I, Boomsma D, Heid IM, Pramstaller PP, Penninx BW, Janssens AC, Wilson JF, Spector T, et al: Loci influencing lipid levels and coronary heart disease risk in 16 European population cohorts. Nat Genet 41: 47-55, 2009.

16. Teslovich TM, Musunuru K, Smith AV, Edmondson AC, Stylianou IM, Koseki M, Pirruccello JP, Ripatti S, Chasman DI, Willer CJ, et al: Biological, clinical and population relevance of 95 loci for blood lipids. Nature 466: 707-713, 2010.

17. Asselbergs FW, Guo Y, van Iperen EP, Sivapalaratnam S, Tragante V, Lanktree MB, Lange LA, Almoguera B, Appelman YE, Barnard J, et al: Large-scale gene-centric meta-analysis across 32 studies identifies multiple lipid loci. Am J Hum Genet 91: 823-838, 2012.

18. Willer CJ, Schmidt EM, Sengupta S, Peloso GM, Gustafsson S, Kanoni S, Ganna A, Chen J, Buchkovich ML, Mora S, et al: Discovery and refinement of loci associated with lipid levels. Nat Genet 45: 1274-1283, 2013.

19. Peloso GM, Auer PL, Bis JC, Voorman A, Morrison AC, Stitziel NO, Brody JA, Khetarpal SA, Crosby JR, Fornage M, et al: Association of low-frequency and rare coding-sequence variants with blood lipids and coronary heart disease in 56,000 whites and blacks. Am J Hum Genet 94: 223-232, 2014.

20. Surakka I, Horikoshi M, Mägi R, Sarin AP, Mahajan A, Lagou V, Marullo L, Ferreira T, Miraglio B, Timonen S, et al: The impact of low-frequency and rare variants on lipid levels. Nat Genet 47 : 589-597, 2015.

21. Lange LA, Hu Y, Zhang H, Xue C, Schmidt EM, Tang ZZ, Bizon C, Lange EM, Smith JD, Turner EH, et al: Whole-exome sequencing identifies rare and low-frequency coding variants associated with LDL cholesterol. Am J Hum Genet 94: 233-245, 2014

22. Helgadottir A, Gretarsdottir S, Thorleifsson G, Hjartarson E, Sigurdsson A, Magnusdottir A, Jonasdottir A, Kristjansson H, Sulem P, Oddsson A, et al: Variants with large effects on blood lipids and the role of cholesterol and triglycerides in coronary disease. Nat Genet 48: 634-639, 2016.

23. Liu DJ, Peloso GM, Yu H, Butterworth AS, Wang X, Mahajan A, Saleheen D, Emdin C, Alam D, Alves AC, et al: Exome-wide association study of plasma lipids in $>300,000$ individuals. Nat Genet 49: 1758-1766, 2017.

24. Hoffmann TJ, Theusch E, Haldar T, Ranatunga DK, Jorgenson E, Medina MW, Kvale MN, Kwok PY, Schaefer C, Krauss RM, et al: A large electronic-health-record-based genome-wide study of serum lipids. Nat Genet 50: 401-413, 2018.

25. Lu X, Peloso GM, Liu DJ, Wu Y, Zhang H, Zhou W, Li J, Tang CS, Dorajoo R, Li H, et al: Exome chip meta-analysis identifies novel loci and East Asian-specific coding variants that contribute to lipid levels and coronary artery disease. Nat Genet 49: 1722-1730, 2017.

26. Spracklen CN, Chen P, Kim YJ, Wang X, Cai H, Li S, Long J, Wu Y, Wang YX, Takeuchi F, et al: Association analyses of East Asian individuals and trans-ancestry analyses with European individuals reveal new loci associated with cholesterol and triglyceride levels. Hum Mol Genet 26: 1770-1784, 2017.

27. Kurano M, Tsukamoto K, Kamitsuji S, Kamatani N, Hara M, Ishikawa T, Kim BJ, Moon S, Jin Kim Y and Teramoto T: Genome-wide association study of serum lipids confirms previously reported associations as well as new associations of common SNPs within PCSK7 gene with triglyceride. J Hum Genet 61: 427-433, 2016.

28. Yamada Y, Sakuma J, Takeuchi I, Yasukochi Y, Kato K, Oguri M, Fujimaki T, Horibe H, Muramatsu M, Sawabe M, et al: Identification of eight genetic variants as novel determinants of dyslipidemia in Japanese by exome-wide association studies. Oncotarget 8: 38950-38961, 2017.

29. Yamada Y, Matsui K, Takeuchi I, Oguri M and Fujimaki T: Association of genetic variants with hypertension in a longitudinal population-based genetic epidemiological study. Int J Mol Med 35: 1189-1198, 2015.

30. Grove ML, Yu B, Cochran BJ, Haritunians T, Bis JC, Taylor KD, Hansen M, Borecki IB, Cupples LA, Fornage M, et al: Best practices and joint calling of the HumanExome BeadChip: The CHARGE Consortium. PLoS One 8: e68095, 2013.

31. Anderson CA, Pettersson FH, Clarke GM, Cardon LR, Morris AP and Zondervan KT: Data quality control in genetic case-control association studies. Nat Protoc 5: 1564-1573, 2010. 
32. Price AL, Patterson NJ, Plenge RM, Weinblatt ME, Shadick NA and Reich D: Principal components analysis corrects for stratification in genome-wide association studies. Nat Genet 38: 904-909, 2006.

33. Leslie R, O'Donnell CJ and Johnson AD: GRASP: Analysis of genotype-phenotype results from 1,390 genome-wide association studies and corresponding open access database. Bioinformatics 30: i185-i194, 2014.

34. Eicher JD, Landowski C, Stackhouse B, Sloan A, Chen W, Jensen N, Lien JP, Leslie R and Johnson AD: GRASP v2.0: An update on the genome-wide repository of associations between SNPs and phenotypes. Nucleic Acids Res 43 (Database Issue): D799-D804, 2015.

35. Warde-Farley D, Donaldson SL, Comes O, Zuberi K, Badrawi R, Chao P, Franz M, Grouios C, Kazi F, Lopes CT, et al: The GeneMANIA prediction server: Biological network integration for gene prioritization and predicting gene function. Nucleic Acids Res 38: W214-W220, 2010.

36. Montojo J, Zuberi K, Rodriguez H, Kazi F, Wright G, Donaldson SL, Morris Q and Bader GD: GeneMANIA cytoscape plugin: Fast gene function predictions on the desktop. Bioinformatics 26: 2927-2928, 2010.

37. Montojo J, Zuberi K, Rodriguez H, Bader GD and Morris Q: GeneMANIA: Fast gene network construction and function prediction for Cytoscape. F1000Res 3: 153, 2014.
38. Shannon P, Markiel A, Ozier O, Baliga NS, Wang JT, Ramage D, Amin N, Schwikowski B and Ideker T: Cytoscape: A software environment for integrated models of biomolecular interaction networks. Genome Res 13: 2498-2504, 2003.

39. Piñero J, Queralt-Rosinach N, Bravo À, Deu-Pons J, Bauer-Mehren A, Baron M, Sanz F and Furlong LI: DisGeNET: A discovery platform for the dynamical exploration of human diseases and their genes. Database (Oxford) 2015: bav028, 2015.

40. Piñero J, Bravo À, Queralt-Rosinach N, Gutiérrez-Sacristán A, Deu-Pons J, Centeno E, García-García J, Sanz F and Furlong LI: DisGeNET: A comprehensive platform integrating information on human disease-associated genes and variants. Nucleic Acids Res 45: D833-D839, 2017.

41. Lempiäinen H, Brænne I, Michoel T, Tragante V, Vilne B, Webb TR, Kyriakou T, Eichner J, Zeng L, Willenborg C, et al: Network analysis of coronary artery disease risk genes elucidates disease mechanisms and druggable targets. Sci Rep 8: 3434, 2018.

This work is licensed under a Creative Commons Attribution-NonCommercial-NoDerivatives 4.0 International (CC BY-NC-ND 4.0) License. 